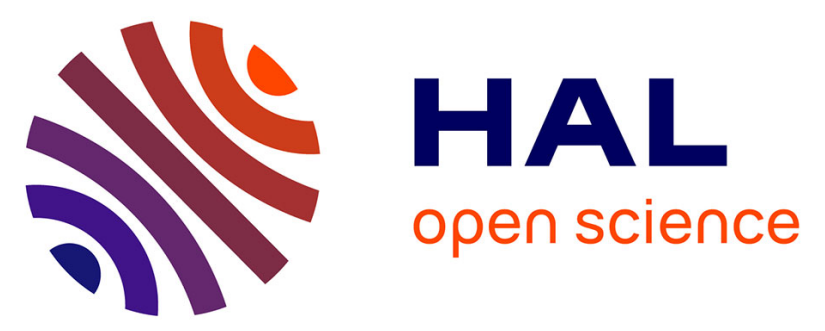

\title{
Solar irradiance observations with PREMOS filter radiometers on the PICARD mission: In-flight performance and data release
}

Gaël Cessateur, Wermer Schmutz, Christoph Wehrli, Julian Groebner, Margit Haberreiter, Matthieu Kretzschmar, Egene Rozanov, Micha Schöll, Alexander Shapiro, Gérard Thuillier, et al.

\section{To cite this version:}

Gaël Cessateur, Wermer Schmutz, Christoph Wehrli, Julian Groebner, Margit Haberreiter, et al.. Solar irradiance observations with PREMOS filter radiometers on the PICARD mission: In-flight performance and data release. Astronomy and Astrophysics - A\&A, 2016, 588, A126 (16p.). 10.1051/0004$6361 / 201527577$. insu-01286003

\section{HAL Id: insu-01286003 https://hal-insu.archives-ouvertes.fr/insu-01286003}

Submitted on 10 Mar 2016

HAL is a multi-disciplinary open access archive for the deposit and dissemination of scientific research documents, whether they are published or not. The documents may come from teaching and research institutions in France or abroad, or from public or private research centers.
L'archive ouverte pluridisciplinaire HAL, est destinée au dépôt et à la diffusion de documents scientifiques de niveau recherche, publiés ou non, émanant des établissements d'enseignement et de recherche français ou étrangers, des laboratoires publics ou privés. 


\title{
Solar irradiance observations with PREMOS filter radiometers on the PICARD mission: In-flight performance and data release
}

\author{
G. Cessateur ${ }^{1,2}$, W. Schmutz ${ }^{1}$, C. Wehrli ${ }^{1}$, J. Gröbner ${ }^{1}$, M. Haberreiter ${ }^{1}$, M. Kretzschmar ${ }^{3}$, E. Rozanov ${ }^{1,4}$, M. Schöll $^{1}$,
} A. Shapiro $^{5}$, G. Thuillier ${ }^{6}$, T. Egorova ${ }^{1,4}$, W. Finsterle ${ }^{1}$, N. Fox ${ }^{7}$, J.-F. Hochedez ${ }^{6,8}$, S. Koller $^{1}$, M. Meftah ${ }^{6}$, P. Meindl ${ }^{9}$, S. Nyeki ${ }^{1}$, D. Pfiffner ${ }^{1}$, H. Roth ${ }^{1}$, M. Rouzé ${ }^{10}$, M. Spescha ${ }^{1}$, R. Tagirov ${ }^{1}$, L. Werner ${ }^{9}$, J.-U. Wyss ${ }^{1}$

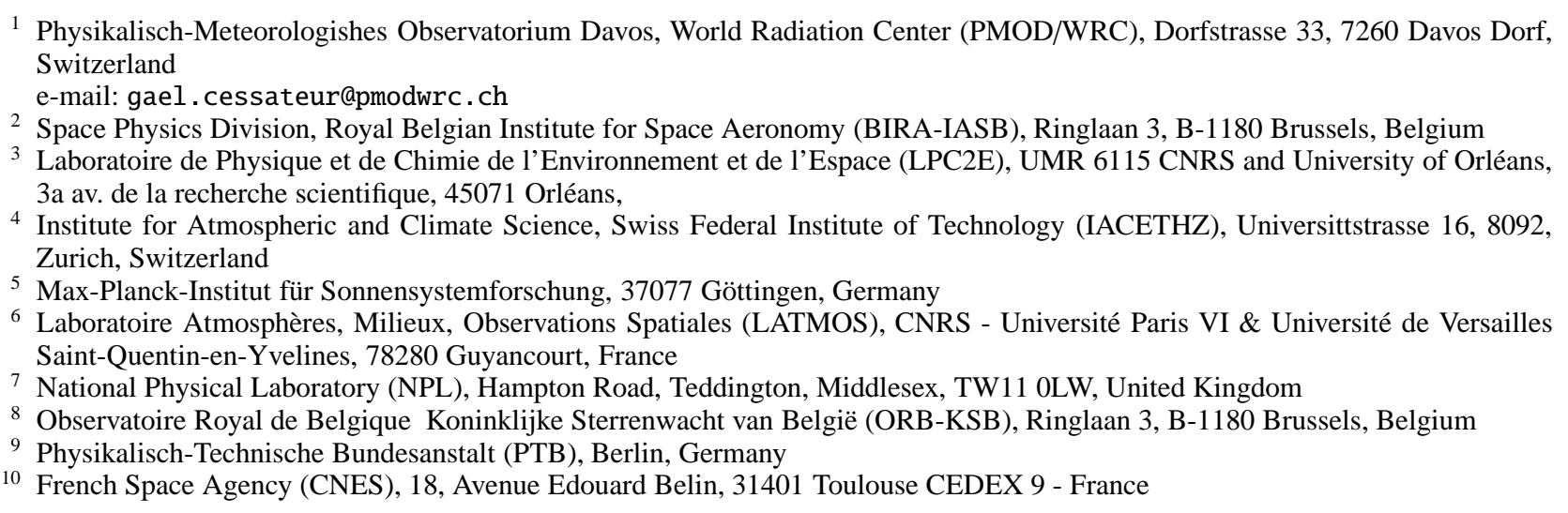

Preprint online version: February 23, 2016

\begin{abstract}
Context. The PREcision Monitoring Sensor (PREMOS) is a solar radiometer on board the French PICARD mission that was launched in June 2010 and decommissioned in April 2014.

Aims. The PREMOS radiometer obtains solar irradiance measurements in specific spectral windows in the UV, visible, and nearinfrared. In this paper, the PREMOS data and calibration methods are presented.

Methods. Using back-up channels, the degradation can theoretically be assessed to correct operational channels. However, a strong degradation within all PREMOS channels requires the application of additional methods, namely using back-up channels and assessing the degradation via a proxy-based model.

Results. The corrected Level 3 PREMOS data are then used in different contexts in order to be validated. First, the signature of the p-mode are retrieved from the PREMOS data. The Venus transit allows us to empirically determine the intrinsic noise level within the PREMOS high cadence data for the visible and near-infrared channels. We then compare the PREMOS data directly to other data sets, namely from the SOLar-STellar Irradiance Comparison Experiment (SOLSTICE) and the Solar Irradiance Monitor (SIM) instruments on board the SOlar Radiation and Climate Experiment (SORCE) spacecraft. Regarding the UV channels, we found an excellent correlation over the lifetime of the PREMOS mission. The ratio between SORCE and PREMOS observations is always less than $1 \%$. Regarding the SSI measurements in the visible and near-infrared, a comparison of short-term variations (i.e. 27-day modulation) shows a rather good correlation by taking into consideration the intrinsic noise within both SIM and PREMOS observations.
\end{abstract}

Key words. Sun: activity - Instrumentation: photometers - Sun: UV radiation - solar-terrestrial relations

\section{Introduction}

The French micro-satellite mission PICARD (Buisson et al. 2006) built by Centre National d'Etudes Spatiales (CNES, France), was successfully launched into a Sun-synchronous dawn-dusk orbit on 15 June 2010. It was then commissioned on October 2010 and decommissioned on April 2014. The scientific goals of the PICARD mission were to investigate the influence of solar forcing on Earth's climate (Thuillier et al. 2006). As a result of the interplay of the different forcing mechanisms, it is clear that further chemistry-climate models (CCM) simulations are needed to quantify the effect of solar variability on the Earth's climate (see e.g. Ermolli et al. 2013). In addition, the PICARD mission was designed to address the physical processes leading to solar irradiance variability by measuring the total solar irradiance (TSI) and solar spectral irradiance (SSI) at specific wavelengths from the middle ultraviolet to near-infrared. The PICARD payload was composed of three experiments including the PREcision Monitoring Sensor (PREMOS) instrument (Schmutz et al. 2009), described in Sect. 2. The SOlar DIameter and Surface Mapper (SODISM) instrument was designed to measure the solar diameter, to monitor the solar asphericity, and to perform helioseismologic observations (Meftah et al. 2014b). The SOlar VAriability PICARD (SOVAP) instrument was devoted to observation of the TSI (Conscience et al. 2011; Meftah et al. 2014a).

PREMOS (Fig. 1) hosted two experiments: one measuring the 
TSI with absolute radiometers and the other measuring the SSI at six wavelengths with different filter radiometers. The key features within both TSI and SSI observations are the 11-year activity cycle and the 27-day rotational modulation. While the TSI variability is less than $0.1 \%$ over the 11 -year solar cycle (Fröhlich 2013), the SSI variability is a strong function of wavelength (see e.g. Fig. 1 from Barthelemy \& Cessateur 2014). Both TSI and SSI measurements are of primary interest for space weather and space climate studies. The planetary climate response to the solar irradiance is sensitive to the 11-year cycle, when considering the Earth (Egorova et al. 2004; Ermolli et al. 2013) as well as planetary objects such as the jovian icy moons (Cessateur et al. 2016a). The 27-day solar modulation also has a strong impact on mesospheric neutral species such as hydroxyl (e.g. Shapiro et al. 2012).

Modelling aside, our current understanding of the solar irradiance variability relies on measurements obtained from space in most cases from an Earth's orbit. Ermolli et al. (2013) gave an overview of the different space instrumentation devoted to SSI observations since the late 1960's. Regular observations of the UV spectral bands $(120-400 \mathrm{~nm})$ were provided by the SOLarSTellar Irradiance Comparison Experiment (SOLSTICE) and Solar Ultraviolet Spectral Irradiance Monitor (SUSIM) instruments aboard the UARS (Upper Atmosphere Research Satellite) spacecraft (Rottman et al. 2004) from 1991 to 2001 and 2005, respectively. Complementary observations of the spectral band between 200 and $400 \mathrm{~nm}$ were also made by the NOAA SBUV (Solar Backscatter UltraViolet) instruments series (Cebula et al. 1998) from 1986 to 2005. Those experiments strongly outline the important variability of the irradiance in the UV. However, the solar cycle variability for wavelengths above $250 \mathrm{~nm}$ still remains unclear because of the long-term instrumental instability. Following these experiments, the SOLSTICE II instrument (Snow et al. 2005), aboard the SOlar Radiation and Climate Experiment (SORCE) spacecraft (Rottman 2005), has been acquiring daily observations of the solar UV irradiance from 180 and $310 \mathrm{~nm}$ since 2003. Merging all of the UV observations into a single composite is a difficult task (see e.g. Schöll et al. 2016). Missing observations can be filled via different statistical methods using solar proxies. However, it has been shown that none of the current solar proxies can properly reconstruct the solar UV irradiance on all timescales (Dudok de Wit et al. 2009), making direct observations of the UV irradiance mandatory for all space weather applications (Lilensten et al. 2008).

Regarding the visible and NIR spectral bands, several instruments have been launched into space to acquire irradiance observations as summarized by Ermolli et al. (2013). Instruments, which have simultaneously conducted observations with PREMOS, include the following: Firstly, the VIRGO-SPM instrument aboard SoHO has been measuring the irradiance time series at $402 \mathrm{~nm}, 500 \mathrm{~nm}$, and $862 \mathrm{~nm}$ since 1996 (Fröhlich et al. 1995). Secondly, the Solar Irradiance Monitor (SIM) instrument (Harder et al. 2005) aboard SORCE has been still acquiring daily spectra from $310 \mathrm{~nm}$ to $2400 \mathrm{~nm}$ since 2003; we perform a direct comparison with PREMOS channels in the visible and near-infrared spectral ranges with these spectra. In addition, the SOLSPEC instrument (Thuillier et al. 2009) aboard the International Space Station (ISS) has been conducting irradiance measurements from $200 \mathrm{~nm}$ to $2400 \mathrm{~nm}$ since 2008 . However, the SOLSPEC data have so far mainly been used to study the 27-day solar modulation during specific observation campaigns as described by Thuillier et al. (2014).
The long-term monitoring of the solar irradiance, however, is a major challenge. Generally, space instrumentation suffers significantly from degradation and signal contamination, which is particularly severe for instruments devoted to SSI observations (BenMoussa et al. 2013). Two methods are usually used to correct the degradation. The first is to rely on redundant systems in which rarely exposed spectral channels are used to periodically correct the degradation of the operational channel. This method is used for most of the radiometers such as SoHO/VIRGO, PICARD/PREMOS, and PROBA2/LYRA (Hochedez et al. 2006; Dominique et al. 2013). The second option is to use reference data, such as periodic rocket launches of a clone instrument, or stable external sources, such as selected stars (in the case of the SOLSTICE I and II instruments) or calibrated lamps (in the case of the SOLSPEC instrument). However, the SSI variability over the long-term (i.e. 11-year solar cycle) is highly uncertain because of the accumulated uncertainty. There are indeed conflicting trends between SIM observations and VIRGO data (Wehrli et al. 2013) and between SSI observations and semi-empirical models, such as SATIRE-S, over the whole spectrum (see Yeo et al. 2014, for a review).

In Sect. 2, the PREMOS instrument is described where the focus is on the strategy of observations as well as a description of the different data levels. Section 3 provides an overview of the PREMOS in-flight performance including the PREMOS first light, the non-solar features which should be extracted from the PREMOS data, as well as the procedures that are to be applied to correct the PREMOS data from degradation. In Sect. 4, a series of tests is performed on the corrected PREMOS data, including a comparison with SORCE/SOLSTICE and SORCE/SIM observations to validate the PREMOS data. Conclusions follow in Sect. 5.

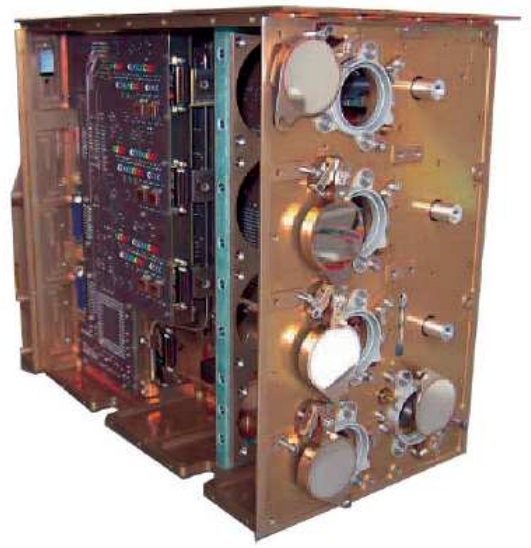

Fig. 1. Assembled PREMOS experiment: SSI radiometers are behind the upper three covers while the two TSI absolute radiometers are located in the lower part. Image provided by Schmutz et al. (2009).

\section{PREMOS instrument description}

The calibration and correction methods for the PREMOS TSI observations was previously discussed by Schmutz et al. (2013). Here, we focus on the PREMOS SSI observations. The SSI instrumentation consists of three heads, named A, B, and C, where each head is equipped with an individual cover and is comprised of four spectral channels. The wavelength characteristics 
of PREMOS are indicated in Table 1. Heads $\mathrm{A}$ and $\mathrm{C}$ are designed to be identical in terms of spectral coverage and filter and detector characteristics. The central wavelengths for Heads A and $C$ are $210 \mathrm{~nm}, 266 \mathrm{~nm}, 535 \mathrm{~nm}$, and $782 \mathrm{~nm}$. Head B is also composed of four channels measuring $215 \mathrm{~nm}$ and $607 \mathrm{~nm}$ with two operational channels and two back-ups. Figure 2 shows the filter characteristics of each channel, together with a synthetic quiet sun spectrum calculated with the COde for Solar Irradiance (COSI; Haberreiter et al. 2008; Shapiro et al. 2010). Table 1 presents a summary of all units and their accompanying channels, their central wavelengths, and their respective FWHM. The visible light and infrared filters are narrowband filters with a filter width ranging from $0.58 \mathrm{~nm}$ to $1.73 \mathrm{~nm}$. The PREMOS UV filters are broadband filters with a full width at half maximum (FWHM) of $20 \mathrm{~nm}$ (for 210 and $266 \mathrm{~nm}$ channels) and $10 \mathrm{~nm}$ (for 215 channels).

The PREMOS UV filters are a combination of two UV filters, one narrowband with one broadband filter to maximise the outof-band rejection. Figure 3 shows the normalised transmittance of the PREMOS filter at $215 \mathrm{~nm}$ from $190 \mathrm{~nm}$ to $1 \mu \mathrm{m}$. Using a solar composite spectrum from the SORCE experiment, an out-of-band rejection of $10^{-3}$ for visible and near-infrared spectral bands is obtained. Similar characteristics were observed for the 210 and $266 \mathrm{~nm}$ channels. Using two combined filters also has the advantage that pinhole pollution is prevented, as it is very unlikely that two pinholes are aligned. The complete filters (i.e. broadband and narrowband) were characterised in-house at Physikalisch-Meteorologisches Observatorium Davos/ World Radiation Center (PMOD/WRC) as solar blind in the visible and near infrared. The SODISM filters, which have been manufactured in the same way as the PREMOS filters, are also solar blind in these spectral ranges.

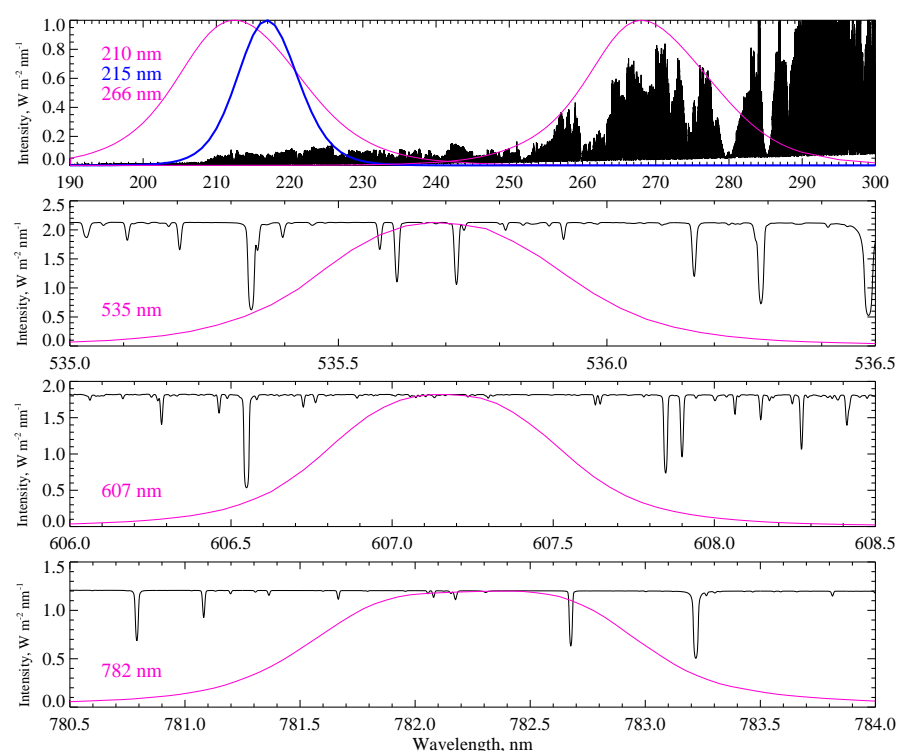

Fig. 2. Bandpasses of the different PREMOS filters, centred at $210 \mathrm{~nm}$, $215 \mathrm{~nm}, 266 \mathrm{~nm}, 535 \mathrm{~nm}, 607 \mathrm{~nm}$, and at $782 \mathrm{~nm}$ along with the COSI spectrum of the quiet Sun (black).

The PREMOS instrument covers an important part of the solar spectral range, from the UV to the near-infrared. The passbands of the filters and their accompanying widths were chosen to maximise their scientific value. The $210 \mathrm{~nm}$ channel, measur-

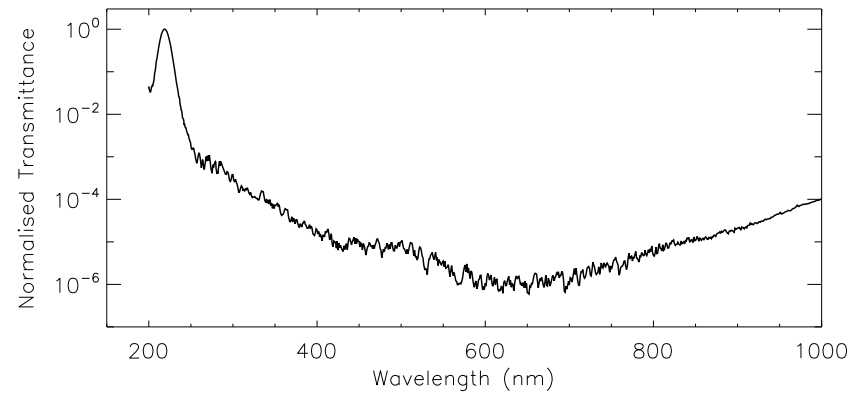

Fig. 3. normalised transmittance for the PREMOS $215 \mathrm{~nm}$ filter from $190 \mathrm{~nm}$ to $1 \mu \mathrm{m}$.

ing the Herzberg continuum, has the same spectral window as that on PROBA2/LYRA (Hochedez et al. 2006; Dominique et al. 2013). However, while LYRA uses experimental diamond detectors (BenMoussa et al. 2009), PREMOS utilises classical silicon detectors for all channels. The UV filters are of primary importance to ozone and molecular oxygen chemistry of planetary atmospheres. The filter design of the UV channels at $215 \mathrm{~nm}$, and the visible-light (VIS) and near-infrared (NIR) channels are similar to that of SODISM (Meftah et al. 2014b). The filter designs were chosen to measure the continuum, by minimizing interference from spectral lines.

The ground calibration of the PREMOS instrument was discussed by Schmutz et al. (2009) and only key values are briefly mentioned here. The filter radiometer channels in the visible and near infrared were characterised in-house at PMOD/WRC. However, the UV channels were calibrated at the PhysikalischTechnische Bundesanstalt (PTB) Berlin. The measurement precision for the UV channels is $1.2 \%$ for the 210 and 215 $\mathrm{nm}$ and $1.4 \%$ for $266 \mathrm{~nm}$ and, thus, is comparable with the SORCE/SOLSTICE instrument for which the measurement precision is about 1-2 \% between 200 and $300 \mathrm{~nm}$ (Snow et al. 2005). Regarding the visible and near-infrared channels, Schmutz et al. (2009) determined a relative uncertainty of about $4 \%$. This is somewhat larger than for the SORCE/SIM instrument, for which the uncertainty budget is less than $2.8 \%$ (Harder et al. 2005).

\subsection{Observation strategy}

As mentioned previously, Heads A and C are similar from a spectral point of view. However, both units are not exposed to the Sun in the same way. This is also the case for the channels in Head B. Those differences in terms of exposure time result from the definition of a redundancy strategy to estimate responsivity loss due to exposure time to sunlight. An attempt to assess instrument degradation was made, in a self-consistent way by, first referring operational measurements to occasional backup operations, and then correcting the backup channel by initial ageing of the operational channel. The strategy is the following:

- Head A is operated continuously at six measurements per minute with an integration time of 9.911 seconds for the normal mode. Head A is in permanent use and is therefore most affected by degradation as discussed in Sect. 3.3. The UV channels have indeed lost more than $99 \%$ of the signal, about 100 days after the start of the mission, making the UV channels mostly blind to any solar signal. 
Table 1. Key characteristics of the PREMOS filters, including centre wavelength and full width at half maximum.

\begin{tabular}{cccccc}
\hline & & Channel 1 & Channel 2 & Channel 3 & Channel 4 \\
\hline Heads A \& C & $\lambda$ & $210 \mathrm{~nm}$ & $266 \mathrm{~nm}$ & $535.69 \mathrm{~nm}$ & $782.26 \mathrm{~nm}$ \\
& FWHM & $20 \mathrm{~nm}$ & $20 \mathrm{~nm}$ & 0.58 & $1.73 \mathrm{~nm}$ \\
\hline Head B & $\lambda$ & $215 \mathrm{~nm}$ & $607.16 \mathrm{~nm}$ & $215 \mathrm{~nm}$ & $607.16 \mathrm{~nm}$ \\
& FWHM & $10 \mathrm{~nm}$ & $0.89 \mathrm{~nm}$ & $10 \mathrm{~nm}$ & $0.89 \mathrm{~nm}$ \\
\hline
\end{tabular}

- Head C, back-up of Head A, is exposed only once per day for three minutes.

- Head B is a self-consistent system with two pairs of identical channels. The first pair is exposed every fourth orbit for one minute, while the second pair is exposed once per week for two minutes.

\subsection{Data products}

PREMOS data are organised in different levels, from 0 to 3 as described below:

- Level 0: Raw Data from PREMOS without any correction applied in units of counts per second.

- Level 1: The data is converted from counts per second to voltage. The data are corrected for temperature variations on the voltage-to frequency converters using ground calibrations. The Earth-Sun distance correction is also applied.

- Level 2A: Data are converted into solar irradiance units (in $\mathrm{W} \mathrm{m} \mathrm{m}^{-2} \mathrm{~nm}^{-1}$ ).

- Level 2B: The temperature sensitivity of the detectors is corrected for the visible and near-infrared channels. Instrumental artefacts within the data are removed. Nonsolar features, as described in Sect. 3.2, are also removed. The degradation is not yet corrected.

- Level 3: Final PREMOS data set. The data are corrected for the degradation using either internal means (i.e. using backup channels) or proxy-based modelling (see Sect. 3.3).

The resulting Level 3 PREMOS data are thus compared with other data sets in Sect. 4.

\section{In-flight performance}

\subsection{PREMOS first light}

Head A was exposed to sunlight for the first time on 27 July 2010 and Head C on 6 August 2010. Head B was exposed on 6 September 2010. Table 2 summarises the first light values obtained with PREMOS for the different spectral channels. These values are compared to the SOLSTICE (level 3, version 15) and SIM (level 3, version 22) instruments aboard SORCE. SOLSTICE and SIM observations for corresponding dates are convolved with the actual transmittance of the PREMOS filters and normalised by the total area under the bandpass curve giving normalised values.

There is an important irradiance offset in the $210 \mathrm{~nm}$ and 215 nm channels compared to SORCE observations. Heads A and C show a difference of $14 \%$ and $20 \%$, respectively, for the irradiance at $210 \mathrm{~nm}$. For the channels 1 and 3 from Head B, observing at $215 \mathrm{~nm}$, the difference is $28.6 \%$ and $17.5 \%$, respectively. This general behaviour can only be explained by degradation of the UV filters prior to the launch. Schmutz et al. (2009) already expressed this concern by noticing that the transmittance of the
UV filters, as evaluated from filter witnesses and different calibration runs, decrease with time. These authors reported a significant $8 \%$ drop of the peak responsivity of the UV filter transmittance between two calibration campaigns six months apart. This responsivity drop exceeds the measurement uncertainty $(1.4 \%$ compared to $8 \%$ ). The $266 \mathrm{~nm}$ channels exhibit different behaviour with an irradiance excess of $5.8 \%$ and $1.2 \%$ for Heads A and C, respectively, compared to SORCE observations. This was not expected, based on ground calibrations performed by Schmutz et al. (2009), where a similar degradation of the 266 $\mathrm{nm}$ filters to the 210 and $215 \mathrm{~nm}$ filters was also observed. One possible explanation is the fact that PREMOS observations are being conducted in space, where the temperature impact on the filter cannot be neglected because there might be insufficient heat dissipation. The filter transmittance can then change, especially in the visible window, allowing more sunlight through the filter and then increasing the resulting measurement. This does not contradict the possibility that the UV filter at $266 \mathrm{~nm}$ also experiences a similar degradation compared to the $210 \mathrm{~nm}$ and $215 \mathrm{~nm}$ filters. Both the temperature impact and degradation processes could happen at the same time. Regarding the filter at $266 \mathrm{~nm}$, the temperature impact might overcompensate the transmittance degradation. This would thus suggest that the degradation of the UV filters at 210 and $215 \mathrm{~nm}$ are more pronounced than initially suggested. Without any further information, it is difficult to make any conclusions on this matter. One solution would be to retrieve each head to perform post-flight characterisation; this is unfortunately not possible. Tests could be conducted using spare instruments, but one main difficulty would be to recreate the same exposure conditions.

For the channels at $535 \mathrm{~nm}$ and $782 \mathrm{~nm}$, it seems that there was also a degradation prior to launch. Heads $\mathrm{A}$ and $\mathrm{C}$ exhibit a difference of $-4.8 \%$ and $-1.7 \%$, respectively, at $535 \mathrm{~nm}$, while this difference is $-4.6 \%$ and $-3.7 \%$ at $782 \mathrm{~nm}$. The Head B channels at $607 \mathrm{~nm}$ exhibit a $+4.3 \%$ and $+5.3 \%$ irradiance increase for channel 2 and 4, respectively. The irradiance offset is within the uncertainty of the calibration, which is thus satisfactory.

Following this evaluation, the PREMOS channels are scaled to the SORCE measurement in terms of absolute values of the irradiance, using the SORCE value measured on the day of the PREMOS first light.

\subsection{Non-solar features}

Along with the degradation processes discussed in Sect. 3.3, the PREMOS data are also affected by undesired features, which have to be removed before we can evaluate the solar signal variations. 
Table 2. normalised values in $\mathrm{W} \mathrm{m}^{-2} \mathrm{~nm}^{-1}$ for the PREMOS first light at 1 astronomical unit, for the UV, visible, and infrared channels. Differences between PREMOS and SORCE observations for the same date are indicated in brackets.

\begin{tabular}{cccccc}
\hline \hline PREMOS & $210 \mathrm{~nm}$ & $215 \mathrm{~nm}$ & $266 \mathrm{~nm}$ & $535.69 \mathrm{~nm}$ & $607.16 \mathrm{~nm}$ \\
\hline Head A & $2.4910^{-2}(-14 \%)$ & & $0.198(+5.8 \%)$ & $1.856(-4.8 \%)$ & $1.138(-4.6 \%)$ \\
Head C & $2.3310^{-2}(-20 \%)$ & & $0.189(+1.2 \%)$ & $1.883(-1.7 \%)$ & $1.121(-3.7 \%)$ \\
Head B (Channels 1 \& 2) & & $2.6410^{-2}(-28.6 \%)$ & & & $1.806(+4.3 \%)$ \\
Head B (Channels 3 \& 4) & & $3.0510^{-2}(-17.5 \%)$ & & & $1.824(+5.3 \%)$ \\
\hline
\end{tabular}

\subsubsection{Temperature effects}

The observations in the visible and near-infrared by PREMOS are very sensitive to orbital temperature variations. Figure 4 shows an example of a full day of quiet-Sun observations with the visible channel at $535 \mathrm{~nm}$ and the temperature response of the PREMOS instrument. The PREMOS filter-detector system is clearly sensitive to temperature variations as indicated in Fig. 4. The PREMOS orbit induces a temperature modulation with a period of $99 \mathrm{~min}$. This was not characterised prior to launch, so the correction is empirical and is directly applied to the data. The interferometric filters used for the visible and near infrared are very sensitive to temperature, which affects the PREMOS measurement. By filtering out this non-solar modulation, the same daily time series becomes more or less a flat line, and any variability is assumed to be of solar origin. For longer timescales, a similar temperature effect has not been identified. As discussed in Sec. 3.3, visible and near-infrared filters are also affected by contamination inducing a similar effect as the temperature variations. It is worth mentioning that the UV channels are not affected by this temperature effect on any timescales. No oscillation patterns have been clearly observed in the UV PREMOS channels. However, the temperature might have an impact on the absolute values of the irradiance as stated in Sect. 3.1. The PREMOS experiment does not provide enough information about this specific issue. Future solar radiometers should address this particular problem.
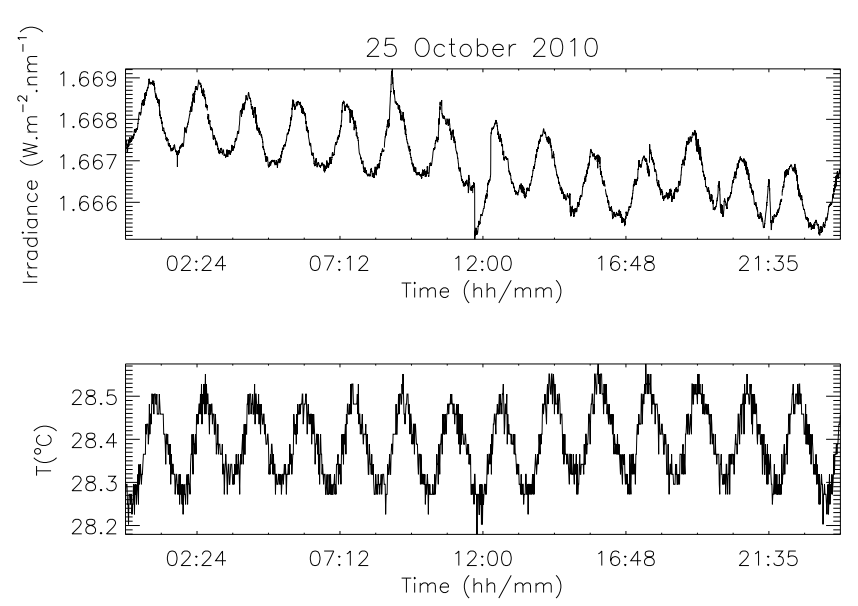

Fig. 4. Daily time series of PREMOS observations at $535 \mathrm{~nm}$ (top panel) and temperature of the Head A on the same day (lower panel).

\subsubsection{South Atlantic Anomaly}

PREMOS signals in the UV channels are particularly affected when transiting the South Atlantic Anomaly (SAA). Secondary electrons generated by high energetic protons can hit the silicon detectors making the signal very noisy. Figure 5 show daily time series for the UV channel at $210 \mathrm{~nm}$, for the early and mid-phase of the mission, which illustrates the evolution of the signal-tonoise ratio. Between these two dates, the PREMOS signal in the UV decreased by more than one order of magnitude. Some SAA contributions are clearly visible, especially around 9 am on 22 June 2011. The PREMOS signal was high enough to prevent the observations from domination by SAA perturbations for the first 150 days of the mission. This is not the case afterwards and high cadence data in the UV spectral range are very sensitive to electronic noise and are no longer reliable. PREMOS data, especially in the UV, must therefore be used with caution. Similar behaviour has also been noticed in the visible and near-infrared channels (see in Fig. 5). Despite the fact that their signal-tonoise ratio is higher than that from the UV channels throughout the PICARD mission, PREMOS signals for the visible and nearinfrared spectral ranges are impacted by the SAA and must also be used with caution.

\subsubsection{Occultation}

The PICARD satellite encountered the Earth's shadow from November to February. When pointing at the Sun, the PREMOS signal is then progressively attenuated until total extinction, resulting in an artificial eclipse. Those eclipses are easily identified as shown in Fig. 6. Such events are not actually removed from PREMOS observations for all data Levels. The PREMOS measurements of irradiance profiles during solar eclipses are of special interest, as the irradiance data acquired during such events allow the centre-to-limb variations (CLV) of the solar brightness to be assessed. The layers of the solar atmosphere can be probed using the solar radiance coming from different disk positions that radiate at different heights. Solar atmosphere models can be tested during these occultation events, as performed with LYRA data (Shapiro et al. 2013) using the Herzberg channel (200-220 $\mathrm{nm})$.

\subsection{Correction for degradation of PREMOS data}

This section presents the different correction procedures that are used throughout the present study to correct the PREMOS data from degradation to produce PREMOS Level 3 data from Level 2B. This step is mandatory and allows us to compare PREMOS data with those from other experiments, as is presented in Sect. 4.

\subsubsection{Heads A \& C}

The PREMOS radiometer experienced a degradation throughout the whole PICARD mission, which was particularly severe for Head A. As shown in Fig. 6, more than $99 \%$ of the signal for both UV channels, at 210 and $266 \mathrm{~nm}$, was lost, while it is more than $50 \%$ for the visible channel. More than $80 \%$ of the signal 

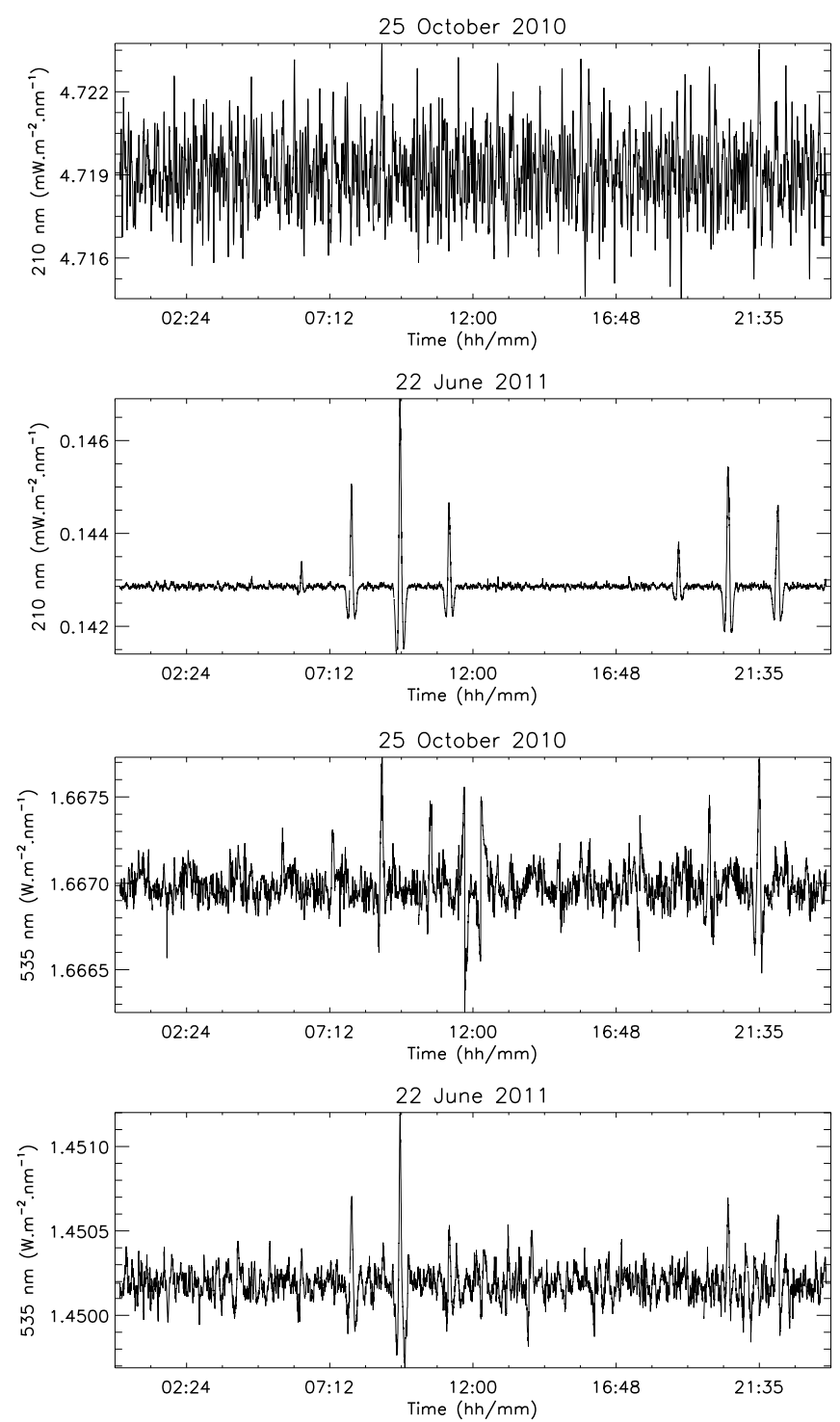

Fig. 5. Daily time series of PREMOS observations at $210 \mathrm{~nm}$ (first two panels) and at $535 \mathrm{~nm}$ (lower panels) on two different days, illustrating different signal-to-noise ratios. The series of periodic peaks correspond to SAA perturbations.

remained for the near IR channel. Head $\mathrm{C}$ suffered from a very pronounced degradation, as shown in Fig. 7. The UV channels at 210 and $266 \mathrm{~nm}$ lost $20 \%$ and $14 \%$, respectively. However, an unexpected behaviour for the channels at $535(-1.1 \%)$ and 782 $\mathrm{nm}(+1.5 \%)$ was encountered. There is indeed some intensity oscillations within the PREMOS data for the visible and nearinfrared channels, and for both Heads A and B.

The degradation is thought to be due to the polymerisation of contaminants on filter surfaces under solar UV exposure. This specific issue is regularly encountered when using similar radiometer designs (see BenMoussa et al. 2013, for a review). The degradation of the UV channels follows a decreasing function. The signal for channels in the visible and the infrared evolves differently over time compared to UV channels, with an intensity oscillation. Unlike the UV filters, visible and infrared filters are interferometric types. The thickness of the contaminant layer therefore plays a major role on the transmittance leading to intensity oscillations. Such behaviour has also been observed

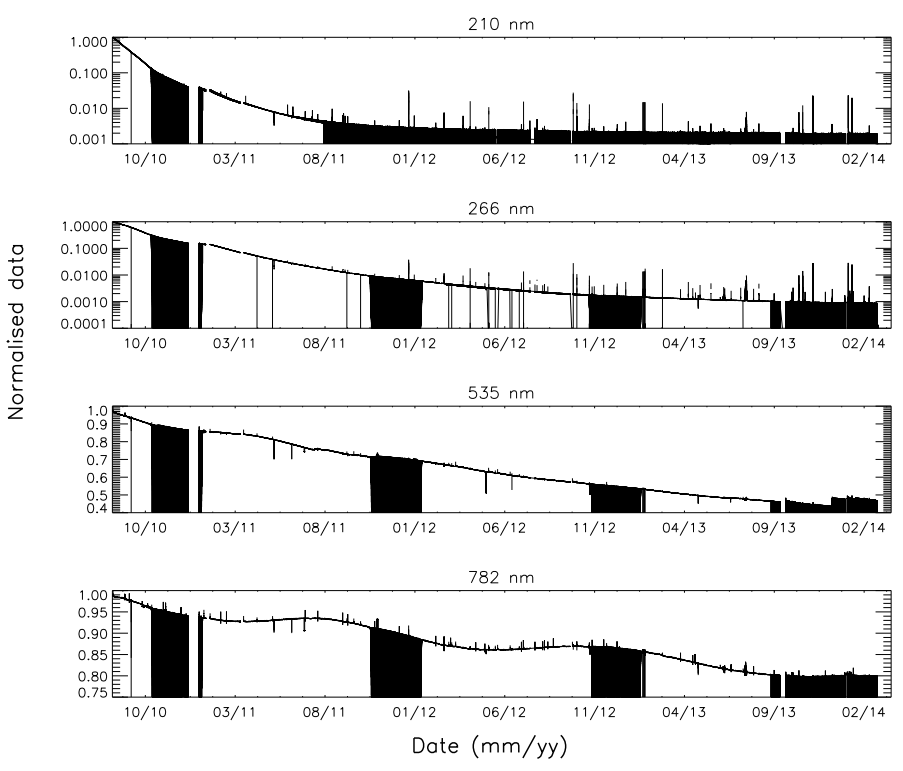

Fig. 6. normalised Level 2B data for the PREMOS Head A, including irradiance observations at $210 \mathrm{~nm}, 266 \mathrm{~nm}$ (both on a logarithmic scale), $535 \mathrm{~nm}$, and $782 \mathrm{~nm}$ (both on a linear scale) from September 2010 to March 2014.
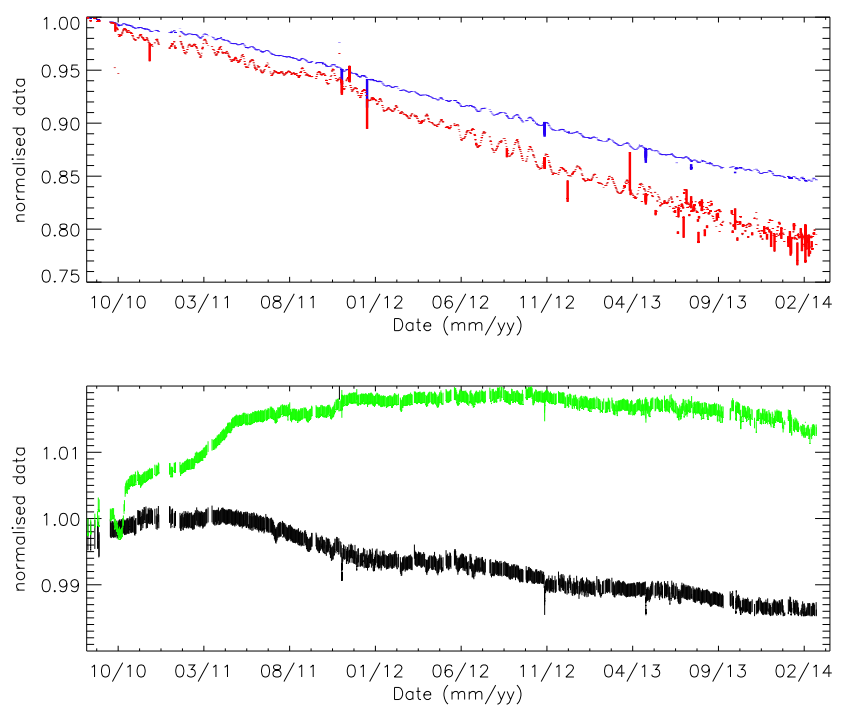

Fig. 7. normalised Level 2B data for the PREMOS Head C, including irradiance observations at $210 \mathrm{~nm}$ and $266 \mathrm{~nm}$ (in red and blue, respectively, in the top figure), and $535 \mathrm{~nm}$ and $782 \mathrm{~nm}$ (in black and green, respectively, in the lower figure) from September 2010 to March 2014.

in the SODISM instrument (see Fig. 27 from BenMoussa et al. 2013), for which the visible and infrared filters share the same key characteristics as the PREMOS filters. Temperature variations of the instrument also have a strong impact on PREMOS observations, while the induced variability ranges from daily to longer timescales as discussed in Sect. 3.2.1. However, a direct comparison with the instrument temperature shows no evident correlation with these oscillations for the channels at 535 and $782 \mathrm{~nm}$. In addition, both visible and near-infrared channels should show correlated oscillations if only temperature variations are responsible, which is not the case (Fig. 6). It is then most likely that the degradation is due to the combination of the polymerisation of contaminants on the surface of filters and the temperature variation of the channels. To better understand 
this unexpected degradation over the PREMOS mission lifetime in the visible and near-infrared channels, we are investigating cross calibration between SODISM and PREMOS observations and will be addressed in a forthcoming paper.

The high cadence data (i.e. from Head A) are very difficult to correct for long-term degradation without a proper reference. Since Head C strongly suffered from degradation in all channels, it is not possible to use it to assess the degradation of Head A. Therefore, high cadence data are mostly interesting for very short-term variability, that is from minutes to hours when the signal-to-noise ratio is very high. However, the UV channels have a very low signal-to-noise ratio roughly 150 days after the beginning of the mission (see Sect. 3.2.2). The degradation is indeed very fast as shown in Fig. 6. Regarding the visible and near-infrared channels from Head A, PREMOS data can be used throughout the PREMOS mission but only to study very shortterm variability.

Regarding the visible and near-infrared channels from Head C, PREMOS data are primarily suitable to study short-term (on the timescale of one solar rotation) variability of solar irradiance by de-trending the PREMOS data. Slow variations, which are understood not to be of solar origin, are thus removed using a Butterworth filter with a cut-off at 81 days.

We now focus on degradation correction of the UV channels from Head C at 210 and $266 \mathrm{~nm}$. The data after May 2013 are not taken into account in the following. Irradiance data at $210 \mathrm{~nm}$ and $266 \mathrm{~nm}$ from Head $\mathrm{C}$ suffer from high instrumental noise after May 2013. There are thus some concerns regarding the data quality about the solar variability. Those data are also discarded for another reason. As a result of malfunctions, PREMOS observations were stopped for several weeks in September 2013. It seems that the UV channels somehow recovered from degradation once normal operation had started again. The reason is that to overcome internal problems, the instrument temperature had to be increased, which probably led to a partial destruction of the contaminant layer. This is clearly visible in the irradiance at $266 \mathrm{~nm}$ (in blue in Fig. 7), where there are two regimes for the degradation before and after September 2013. However, the key problem is the fact that PREMOS data at $210 \mathrm{~nm}$ and $266 \mathrm{~nm}$ are noisy after May 2013. The first approach, which has been used to infer the degradation in the UV, was to compare Head A with Head C. The time exposure is an important parameter and the first approach relies on comparing the initial Sun exposure for both Heads A and C. However, the initial ageing was different, which implies that the degradation does not only depend on the time exposure.

The nature of the degradation is considered a polymerisation of carbon contaminant on top of the filters induced by solar UV photons (BenMoussa et al. 2013; Meftah et al. 2014b). In our case, both the amount of solar UV photons and carbon concentration are the two primary parameters that constrain the degradation. Since the degradation of the UV channels for Head C does not reach an asymptotic limit (see Fig. 7), we assume that the carbon concentration is not a limiting factor here. We thus defined a UV dose using the Lyman $\alpha$ index, which should reflect the number of solar UV photons received by the PREMOS channels from Head C. We first compute daily average values for irradiance at 210 and $266 \mathrm{~nm}$. Each PREMOS measurement represents nearly 10 seconds of exposure. The daily time exposure can thus be calculated and multiplied by the Lyman $\alpha$ index value of the corresponding day. This gives the UV dose function (expressed in $10^{11} \times$ photons. $\mathrm{cm}^{-2}$ ), which estimates the number of solar UV photons that were received by the PREMOS channels.

Figure 8 shows the daily average value at 210 and $266 \mathrm{~nm}$ from PREMOS according to the UV dose. The degradation trend can then be fitted as a function of the UV dose, $1 /\left(\mathrm{a}+\mathrm{bT}_{U V}\right)$, where $\mathrm{a}$ and $\mathrm{b}$ are the coefficients of the regression and $\mathrm{T}_{u v}$ is the UV dose. The PREMOS data at 210 and $266 \mathrm{~nm}$ are now corrected and ready to be compared with other irradiance data sets (see Sect. 4.2).
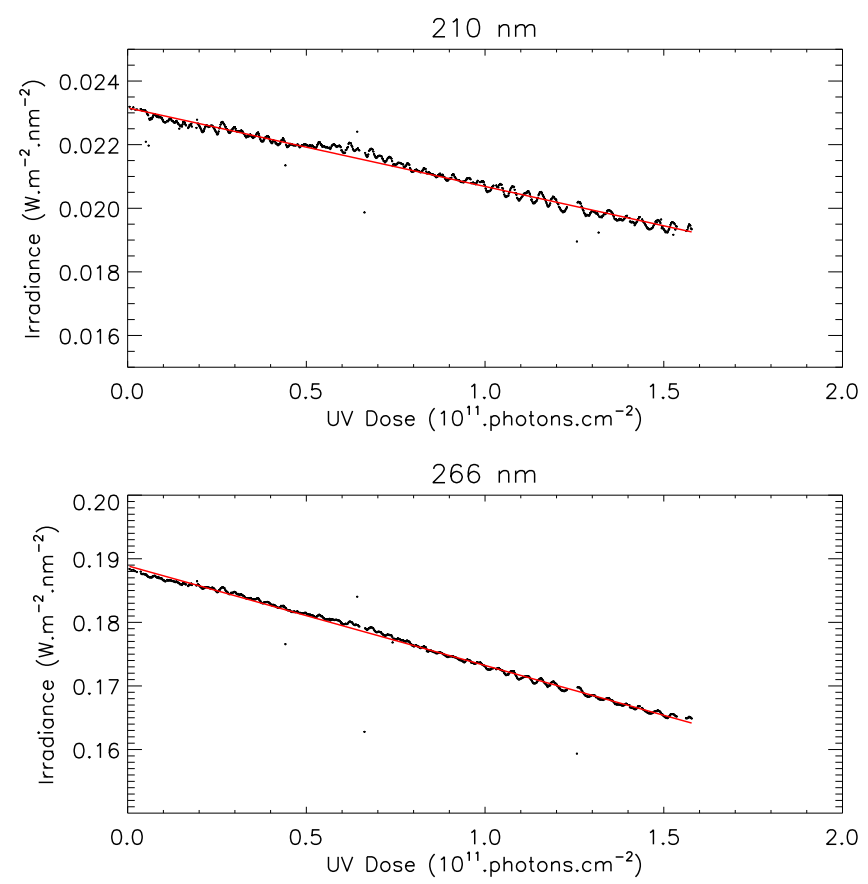

Fig. 8. Daily average irradiance value from Head C at $210 \mathrm{~nm}$ (top panel) and at $266 \mathrm{~nm}$ (lower panel) according to the UV dose. The linear regression fit is also plotted (in red).

\subsubsection{Head B}

Head B, and the strategy which lies behind it, is now considered. As a reminder, Channels 1 and 2 are exposed every fourth orbit for one minute, while Channels 3 and 4 are exposed once per week for two minutes. Figure 9 presents the Level 2 time series for the Head B Channels, including the $215 \mathrm{~nm}$ and 607 nm observations. The curves of Channels 1 and 3 presented here in Fig. 9 have not yet been corrected for degradation, but have been corrected for numerous observational artifacts. Originally, the observational strategy was to assume that Channels 2 and 4 from Head B were unaffected by the degradation. While it is more likely to be true for the $215 \mathrm{~nm}$ Channel (upper panel), this is clearly not the case for Channel 4 at $607 \mathrm{~nm}$.

Both observations at $607 \mathrm{~nm}$, i.e. Channels 2 and 4 from Head $\mathrm{B}$, have indeed suffered from degradation with a signal loss of about $1 \%$ and less than $0.3 \%$, respectively. The intensity oscillations, already noted for irradiance observations at 535 and 782 $\mathrm{nm}$, also affect both visible Channels from Head B. However, 
there is a more pronounced correlation with the temperature, unlike the other visible and near-infrared channels. Because of its degradation, Channel 4 cannot be used as a back-up channel to assess the degradation of Channel 2 from Head B. The temperature dependence can still be corrected, but the contamination layer, which grows with time and impacts the overall transmittance, requires more information than would be available to fully correct the irradiance at $607 \mathrm{~nm}$. Regardless of this, visible data from Head B can actually be used to assess the variability over the short-term. However, numerous data gaps within irradiance measurements from Channel 2 can indeed lead to different degradation regimes, as clearly visible in June 2012. This prevents us from having an overall picture of even the short-term variability (i.e. modulation at 27 days). To overcome this issue, the data from Channel 2 are first scaled against the data from Channel 4. The resulting time series is then more homogenous in terms of degradation. Temperature effects and degradation are then removed by smoothing out the resulting irradiance time series with a Butterworth filter with a cut-off at 81 days. However, all information about long-term variability is lost in this case, leaving only short-term variability. Cross-comparisons between PREMOS channels observing at $535 \mathrm{~nm}$ and $607 \mathrm{~nm}$ are thus performed. This final step allows us to identify daily irradiance values, which do not share the same coherency, and can thus be removed.

Regarding UV observations from Head B at $215 \mathrm{~nm}$, we apply the strategy described in Sect. 2.1 to correct the more exposed channel using only the back-up channel. Channel 1 from Head B has lost roughly $25 \%$ of its signal. Channel 3 is assumed to be unaffected by the degradation. Measurements from Channel 1 are then corrected for its intrinsic degradation by scaling those data against the observations from Channel 3. We applied no further corrections, except for well-known instrumental artifacts. The motivation here is to fully test the redundancy strategy and to figure out for how long this strategy can be applied without any additional degradation correction. We proceed to validate the Level 3 PREMOS data by comparing them to other data sets in the next section.

\section{Validation of the PREMOS data}

\subsection{High cadence data}

\subsubsection{Helioseismology}

With one measurement every 10 seconds for Head A, the PREMOS data set has the advantage of a good time resolution compared to the timescales of solar oscillations. However, as discussed previously, continuous exposure has the disadvantage of leading to a very strong degradation. The PREMOS data from Head A is useful to assess the variability over the very shortterm, from minutes to hours. The first step is to de-trend the data to remove any long-term effects, i.e. the 27-day solar modulation cycle and longer trends. After these corrections, PREMOS data can be used in a helioseismology context. Figure 10 shows the power spectrum for irradiance at $782 \mathrm{~nm}$. We combined $40 \mathrm{sub}$ series, each from one day (earlier days of the mission), to smooth out the instrumental noise. Acoustic oscillations or P-mode have frequencies that are greater than $1 \mathrm{mHz}$ and are very strong between 2 and $4 \mathrm{mHz}$ (Corbard et al. 2013). Such a signature is clearly visible in the $782 \mathrm{~nm}$ channel. We obtained a similar result for the $535 \mathrm{~nm}$ channel. We also observed P-modes with the UV channels at 210 and $266 \mathrm{~nm}$. However, the resulting power
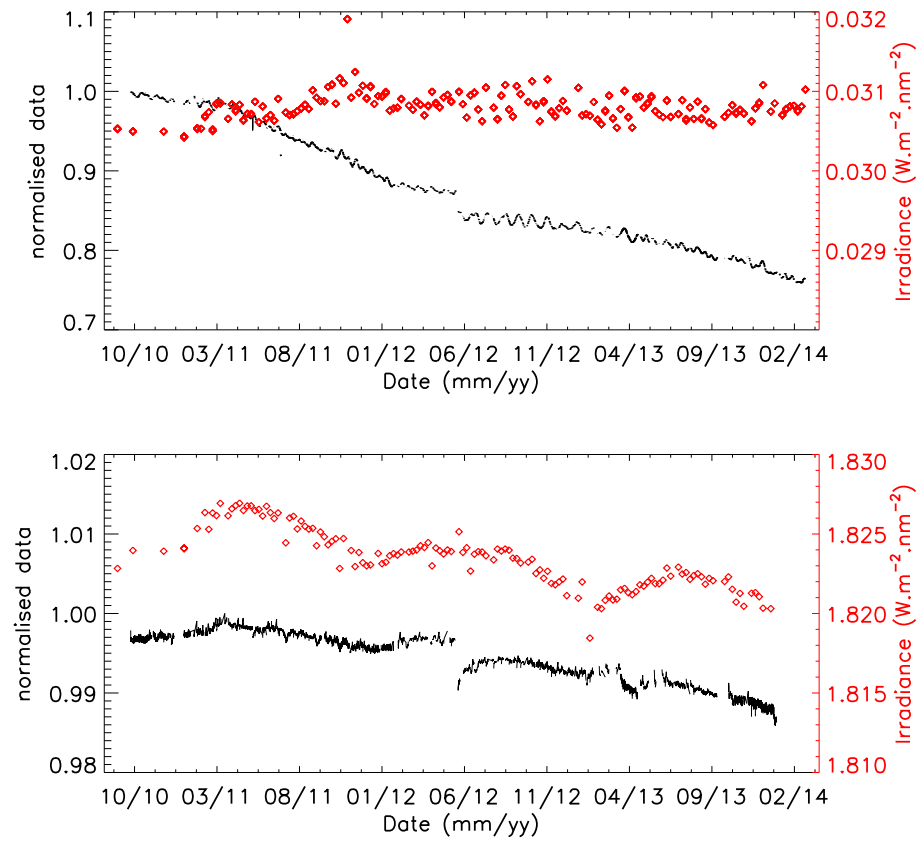

Fig. 9. Level 2 data for the Head B, including irradiance observations at $215 \mathrm{~nm}$ and $607 \mathrm{~nm}$ from September 2010 to March 2014. Channels 1 and 2 are normalised (in black), while Channels 3 and 4 are in irradiance units (in red).

spectrum is much noisier than those we obtained for the visible and infrared channels, which is probably because of a lower signal-to-noise ratio.

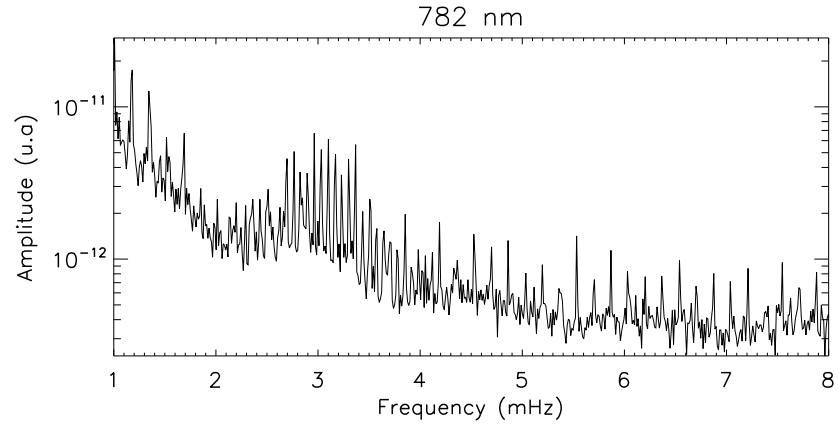

Fig. 10. Power spectrum density at $782 \mathrm{~nm}$ as observed by the PREMOS Head A.

\subsubsection{Solar flare events}

High cadence SSI data might also display signatures of solar flares. For example, the LYRA instrument has been used used to characterise the flare signal in Lyman $\alpha$ (Kretzschmar et al. 2013). However, no clear signature has been detected within PREMOS observations from the UV spectral range to nearinfrared, even for the most important events that occurred during the PICARD mission. Superposed epoch analysis has been able to detect solar flares in TSI and SSI measurements where the flare contrast is low. With this technique, flares have been detected in TSI observations from SoHO (Kretzschmar et al. 2010), and the blue, green, and red channels of the VIRGO/SPM instrument (Kretzschmar 2011), but also using the full solar 
GOLF/SoHO data (Cessateur et al. 2010). We tried to detect flare signatures, however, the PREMOS mission was not long enough and/or the signal-to-noise ratio of the relevant channels decreased too quickly to reveal flare signatures with this technique.

\subsubsection{Venus transit}

During the June 2012 Venus transit, irradiance time series were acquired by Head A from PREMOS. Figure 11 shows the high cadence irradiance at 535 and $782 \mathrm{~nm}$, where an irradiance decrease of about $1100 \mathrm{ppm}$ is caused by the Venus transit. For the sake of comparison, the PREMOS TSI measurement is also shown (in black). By comparing the overall shape of the transit curve, we conclude that the centre-to-limb variation of the solar disk is practically identical for the two wavelengths 535 and $782 \mathrm{~nm}$, as well as for TSI. On a shorter timescales (TSI has two minute time resolution) the three channels share similar signatures; for example an irradiance drop just after midnight. Nevertheless, the correlation coefficient between irradiance measurements is surprisingly low during the Venus transit $\left(\mathrm{R}^{2} \leq 0.3\right)$. A noise level within PREMOS channels at 535 and $782 \mathrm{~nm}$ for Head A can thus be estimated by taking $2 \times \sigma_{\text {PREMOS }}$, where $\sigma_{\text {PREMOS }}$ is the standard deviation of the irradiance measurement during the Venus transit. By doing this, a noise level of about $139 \mathrm{ppm}$ and $157 \mathrm{ppm}$ can be estimated for the Head A channels at $535 \mathrm{~nm}$ and $782 \mathrm{~nm}$, respectively.

No signature of the Venus transit has been detected for the PREMOS UV channels, probably because the signal-to-noise ratio was already too low for both UV channels at 210 and $266 \mathrm{~nm}$ in June 2012.

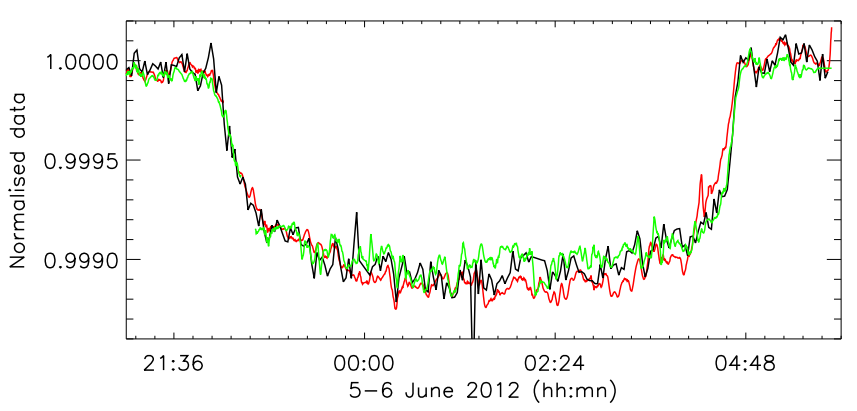

Fig. 11. Transit of Venus from 6 June 2012 as measured with PREMOS at $535 \mathrm{~nm}$ (in red) and at $782 \mathrm{~nm}$ (in green). The PREMOS TSI is also plotted (in black).

\subsection{Solar irradiance variability}

In order to validate the variability of the PREMOS data for shortand long-term variability, both internal comparisons between the UV PREMOS channels and comparisons to SORCE observations are analyzed. We consider data taken from instruments aboard SORCE, namely the SOLSTICE instrument (Level 3, version 15, from 180 to $310 \mathrm{~nm}$ ) and the SIM instrument (Level 3 , version 22). We obtained the daily spectral measurements used here from the LISIRD archive ${ }^{1}$. We use these data without the application of further corrections. Any artifact in the in-

\footnotetext{
1 http://lasp.colorado.edu/lisird/
}

put data affects the simulated outputs when convolving SORCE observation with PREMOS channels. When focusing only on short-term variability for the visible and near-infrared spectral ranges, slow variations are removed using a Butterworth filter with a cut-off at 81 days.

\subsubsection{Comparison between PREMOS UV channels}

We investigate the comparisons between irradiance in the UV as measured by PREMOS. It is well known that the SSI shows a remarkable coherency, which is manifested by a similar time evolution of the irradiance observed at different wavelengths and spectral bands (Dudok de Wit et al. 2005; Cessateur et al. 2011). This high correlation is due to the strong dependence of solar irradiance variability on the magnetic field (Domingo et al. 2009). It can then be expected that a linear relationship between all UV PREMOS channels is observed. As a first test for the UV channels, key values of the relationship between UV channels are provided. Figure 12 shows the irradiance at $215 \mathrm{~nm}$ and at 266 $\mathrm{nm}$ plotted against the irradiance at $210 \mathrm{~nm}$. The resulting correlation coefficients are 0.86 and 0.87 , respectively. When considering the number of points here, $N=690$; this is an excellent correlation for both curves based on the Pearson definition. The 2 -sigma interval is also plotted and the cloud of points are seen to be well within those boundaries. The PREMOS data at 215 and $266 \mathrm{~nm}$ from September 2010 to May 2013 can be expressed with respect to the irradiance at $210 \mathrm{~nm}$ by a linear relationship $a+b \times I_{210}$. Table 3 shows the key values of the linear regression as well as the standard error values. As a reminder, we note that the absolute irradiance values of the PREMOS data used here have been set to SORCE observations.
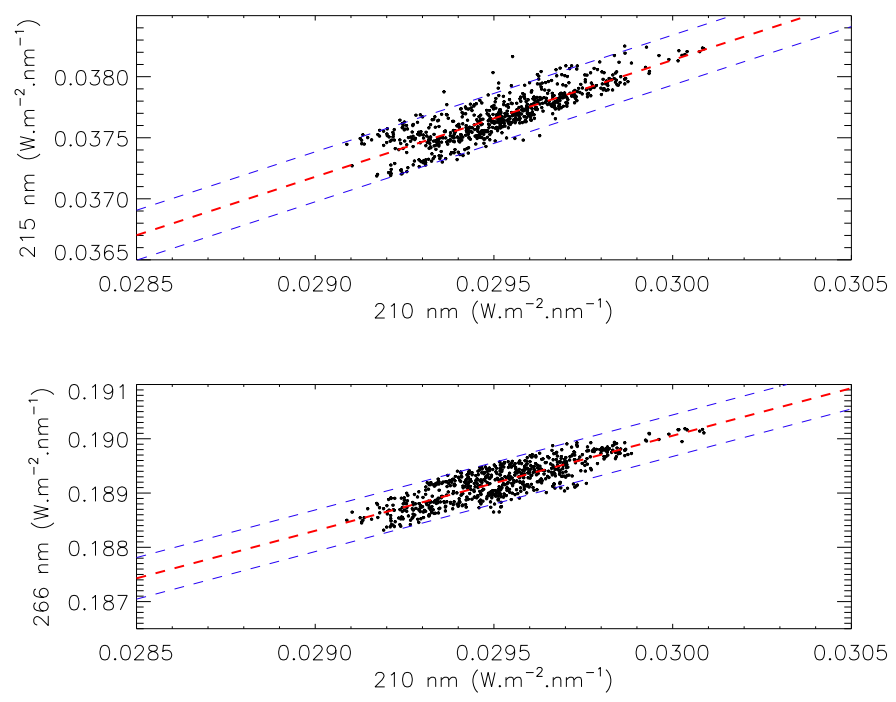

Fig. 12. Irradiance at $215 \mathrm{~nm}$ plotted against the irradiance at $210 \mathrm{~nm}$ (top figure), and irradiance at $266 \mathrm{~nm}$ plotted against the irradiance at $210 \mathrm{~nm}$ (lower figure). The regression lines are shown (red dotted lines), as well as the 2- $\sigma$ intervals (blue dotted lines).

\subsubsection{Comparisons with SORCE observations}

We compare the Level 3 PREMOS data and SORCE observations. First, the irradiance at $210 \mathrm{~nm}, 215 \mathrm{~nm}$, and $266 \mathrm{~nm}$ are compared without de-trending the data. Both short-term (i.e. so- 
Table 3. Key values of the linear relationship between channels at 210, 215 , and $266 \mathrm{~nm}$ as measured by PREMOS. The units of $a$ and $\sigma_{\text {err }}$ are $\mathrm{W} \mathrm{m}{ }^{-2} \mathrm{~nm}^{-1}$.

\begin{tabular}{ccccc}
\hline $\mathrm{a}+\mathrm{b} \times \mathrm{I}_{210}$ & $\mathrm{R}$ & $\mathrm{R}^{2}$ & $\sigma_{\text {err }}$ & $\frac{\sigma_{\text {err }}}{\left\langle\mathrm{I}_{\mathrm{i}}\right\rangle}(\%)$ \\
\hline $\mathrm{I}_{215}=0.011+0.893 \times \mathrm{I}_{210}$ & 0.867 & 0.753 & $110^{-4}$ & $0.27 \%$ \\
\hline $\mathrm{I}_{266}=0.127+2.04 \times \mathrm{I}_{210}$ & 0.869 & 0.756 & $2.210^{-4}$ & $0.12 \%$ \\
\hline
\end{tabular}

lar modulation induced by the rotation) and longer term (i.e. variability over the PREMOS lifetime) are thus compared. Then visible and near-infrared channels are also compared, but only for short-term modulation.

\section{UV spectral range}

Figure 13 shows the direct comparison between the PREMOS and SOLSTICE observations at $210 \mathrm{~nm}$ (top panel) as well as the ratio PREMOS over SOLSTICE (lower panel) for the period between September 2010 to May 2013. The ratio indicates that both data sets agree within $1 \%$. The absolute scale is based on SOLSTICE observations. There is a rather good correlation between both data sets with a Pearson correlation coefficient, $\mathrm{R}$, of $0.85\left(R^{2}=0.72\right.$, see Table 4$)$. The main discrepancies arise from data after November 2012, where there is a difference of about $0.8 \%$ on the absolute value. However, the variability in both data sets is very similar. By considering data prior to November 2012, the correlation coefficient increases to $\mathrm{R}=0.93\left(\mathrm{R}^{2}=0.87\right)$. For the irradiance at $210 \mathrm{~nm}$, the correction procedure using a UV dose based method leads to a difference of less than $0.5 \%$ for the period between October 2010 and November 2012. After November 2012, an uncorrected trend leads to a difference of about $0.8 \%$ compared to the SOLSTICE data.
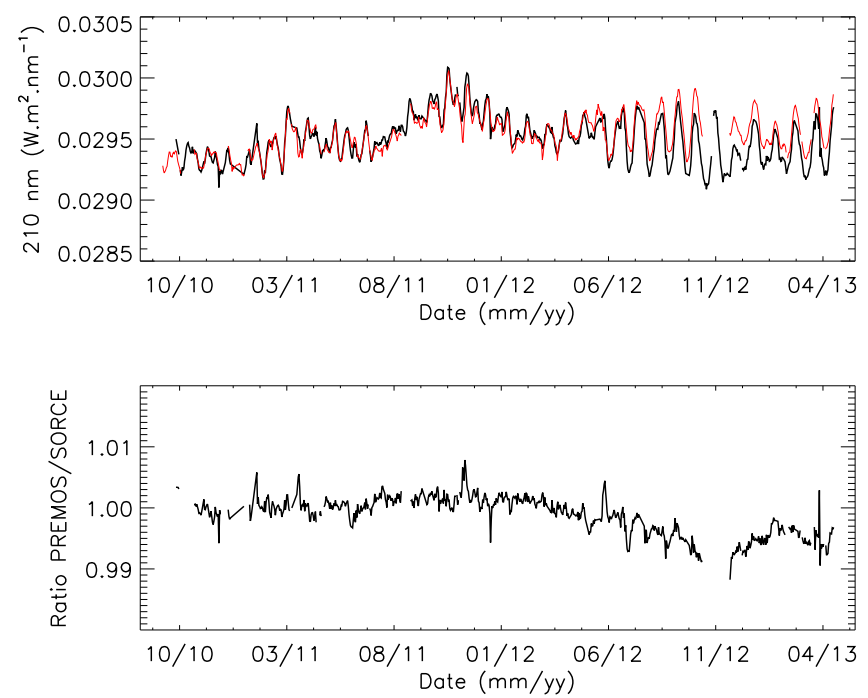

Fig. 13. Level 3 PREMOS data for $210 \mathrm{~nm}$ (in black) and convolved SOLSTICE observations with the PREMOS filter at $210 \mathrm{~nm}$ (in red) from September 2010 to May 2013 (top figure). The PREMOS to SOLSTICE ratio is also indicated (lower figure).

Figure 14 shows the irradiance at $215 \mathrm{~nm}$ as seen by PREMOS and SOLSTICE along with their ratio. As a reminder, we cali- brated the PREMOS channel at $215 \mathrm{~nm}$ by internal means, using the back-up channel from Head B. There is a good correlation coefficient between the two data sets, where $\mathrm{R}=0.91\left(\mathrm{R}^{2}=\right.$ 0.84). The ratio obtained with the SOLSTICE data version 13 is also indicated in Fig. 14 for comparison (in green). Better agreement is found when using the version 15: The independent and internally calibrated PREMOS observation confirms the irradiance variability observed by SORCE/SOLSTICE for this spectral range from September 2010 to August 2013. Interestingly, two independent instruments converge towards the same variability. The underlying observation strategy for Head B, using only one back-up channel exposed once per week, is successful for assessing the irradiance variability in the UV spectral range. After August 2013, it is difficult to reach any conclusions without other observational data sets for comparison. The PREMOS 215 channel could be used to fill the irradiance gap from SORCE from August 2013 to February 2014 using various statistical methods and comparisons with solar modelling. This will be addressed in a forthcoming paper.
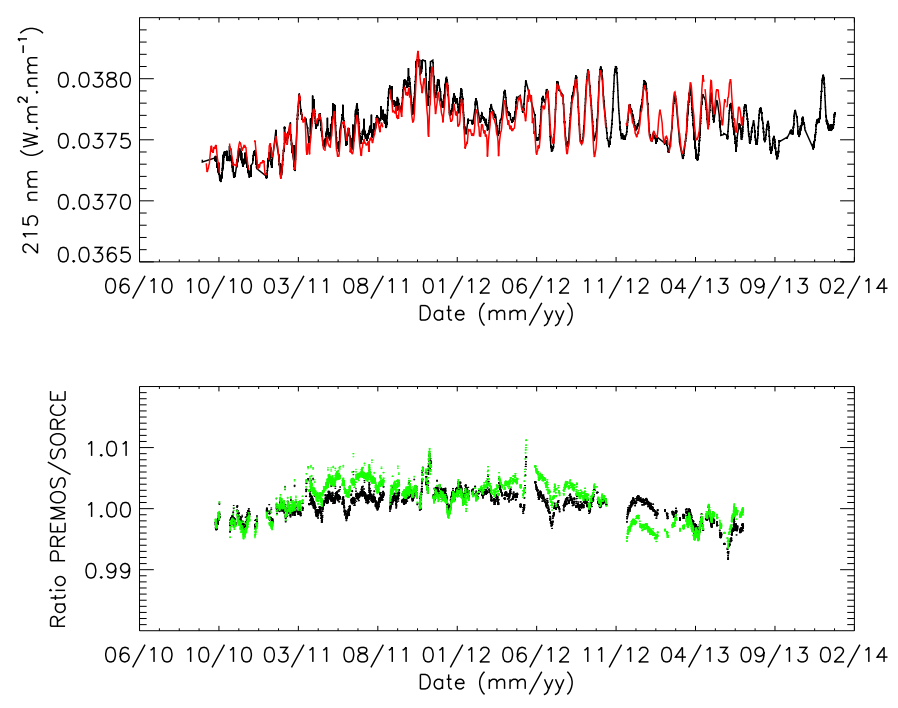

Fig. 14. Same as in Fig. 13 except for irradiance at $215 \mathrm{~nm}$. The PREMOS to SOLSTICE ratio is shown using both versions 13 (in green) and 15 (in black) of the SOLSTICE data.

Figure 15 shows the irradiance at $266 \mathrm{~nm}$ as seen by PREMOS and SOLSTICE, along with their ratio. First, the SORCE data are noisier than for the previous two channels. This could partially explain the correlation coefficient of about $R=0.67\left(R^{2}=\right.$ $0.44)$. Their ratio suggests good overall agreement with a difference less than $0.5 \%$ for the whole period from September 2010 to April 2013. Because of the internal noise of the SORCE observations, the PREMOS data at $266 \mathrm{~nm}$ could be used as a reference for the period considered in Fig. 15.

Regarding the correction of 210 and $266 \mathrm{~nm}$ channels, we used the Lyman $\alpha$ index as proxy. We also used other proxies, such as the F10.7 or the magnesium index (Mg II), and found similar results with a 1-2\% relative difference. Better agreement has, however, been found with SOLSTICE/SORCE data when using the Lyman $\alpha$ index. The level 3 version of PREMOS data for the 210 and $266 \mathrm{~nm}$ channels are thus not fully corrected independently, but are in good agreement with the SOSLTICE/SORCE 
Table 4. Values of $\mathrm{R}$ and $\mathrm{R}^{2}$ (in brackets) for the $\mathrm{UV}$ channels between PREMOS and SORCE observations.

\begin{tabular}{cccc}
\hline & $210 \mathrm{~nm}$ & $215 \mathrm{~nm}$ & $266 \mathrm{~nm}$ \\
\hline PREMOS vs SORCE & $0.85(0.72)$ & $0.91(0.84)$ & $0.67(0.44)$ \\
\hline
\end{tabular}

data version 15 . The channel at $215 \mathrm{~nm}$, however, has been corrected by internal means, completely independent of any other data.
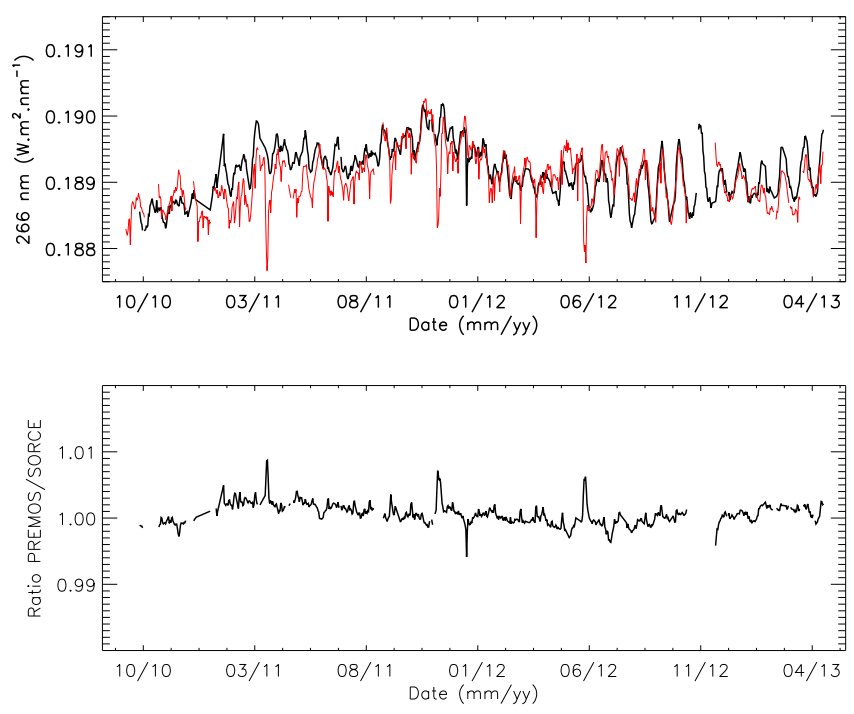

Fig. 15. Same as Fig. 13 except for irradiance at $266 \mathrm{~nm}$.

\section{Visible and near-infrared spectral ranges}

In the following, the comparisons between PREMOS and SIM observations at $535 \mathrm{~nm}, 607 \mathrm{~nm}$, and $782 \mathrm{~nm}$ are discussed. The solar irradiance variability in this part of the spectrum is much smaller than for the UV spectral range regardless of the considered timescales (Ermolli et al. 2013). The signal-to-noise ratio is also lower than for the PREMOS UV channels. It should be noted that only the short-term variability is discussed here, since since we removed the long-term effects (solar signal and degradation).

Figure 16 shows the irradiance at $535 \mathrm{~nm}$ as observed by PREMOS compared to SIM observations. In order to make the figure clearer, only the July 2011 to July 2012 period is shown. The full time series is given in Annex A. As a reminder, we completed the analysis of the PREMOS data over the full time series. From a direct comparison with the normalised data (top panel), both data sets show an excellent correlation during sunspot passages (indicated by thin lines). Except for two cases where SIM observations are probably affected by some outliers, the difference between PREMOS and SIM irradiance is generally close to zero for sunspot passages. Over the full PREMOS mission, the correlation coefficient between PREMOS and SIM observations at $535 \mathrm{~nm}$ is $\mathrm{R}=0.63$. The correlation is rather low because of the intrinsic noise level within PREMOS and SIM observations.

Looking at the difference as well as the resulting probability den-
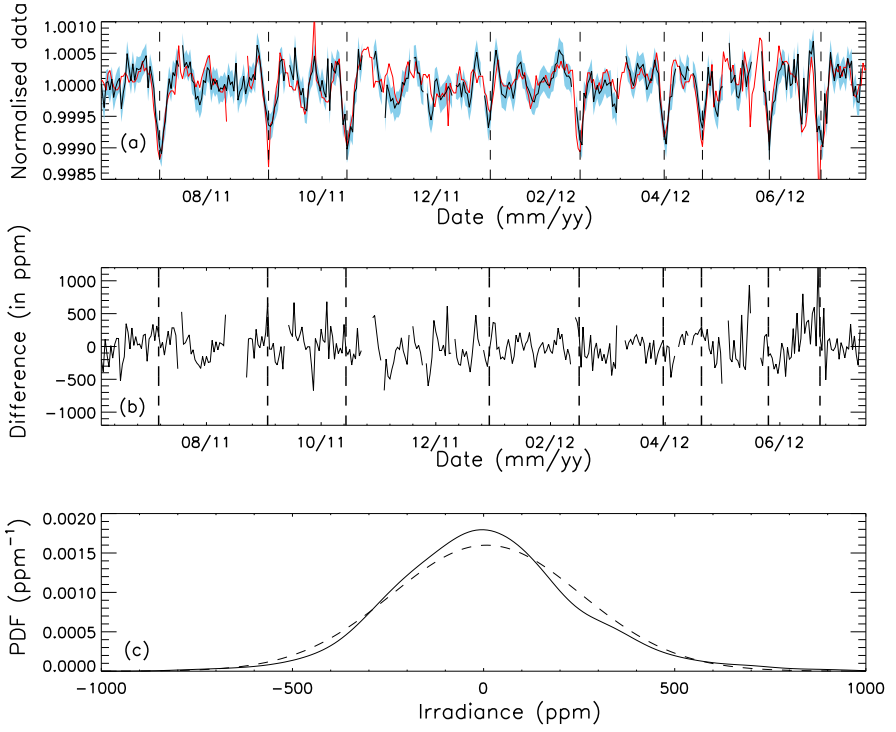

Fig. 16. Short-term variability of the irradiance at $535 \mathrm{~nm}$ as observed by PREMOS (in black) and by SORCE (in red) from July 2011 to July 2012 (panel (a)). The difference (in ppm) between PREMOS and SORCE is also plotted (panel (b)) for the corresponding period. Thin vertical lines indicate the passage of a sunspot. Panel (c) shows the probability density function (PDF) of the difference between both instruments (solid line) along with the respective Gaussian fit (dashed line).

sity function (PDF) could give us more information about the quality of the data. The PDF describes the normalised likelihood of a specific deviation of the data values from the most probable value, where the most probably value is the value where the PDF has its maximum. The probability to obtain a difference of $\pm 0.5 \mathrm{ppm}$ is 0.0015 (see Fig. 15(c)). To obtain a continuous PDF from our discrete data set, namely the approximately 800 measurements, we use a Gaussian kernel density estimator. The crucial parameter for the Gaussian kernel density estimator is the bandwidth selection, which corresponds to the window width of histograms. Scott's Rule (Scott 1992) is used here, which estimates the bandwidth $w$ based on the number of points $n$ and the dimension $d$ as follows:

$w=n^{-1 /(d+4)}$.

By taking into account that the noise in SIM measurements is about 150 ppm (Harder et al. 2009), the noise level of PREMOS data can be estimated by the following equation:

$\sigma_{\text {PREMOS }}=\sqrt{\sigma_{\text {Total }}^{2}-\sigma_{\text {SIM }}^{2}}$,

where $\sigma_{\text {Total }}$ is the sigma deduced from the PDF of the difference between PREMOS and SIM observations, which gives an estimate of the combined noise level. Table 5 presents the $\sigma_{\text {PREMOS }}$ noise level within PREMOS observations. Figure 16 also shows an envelope representing the appropriate uncertainty estimate, i.e. $210 \mathrm{ppm}$. When considering this intrinsic noise level within PREMOS data, SIM observations are generally in good agreement with PREMOS observations at $535 \mathrm{~nm}$, however, their difference is sometimes significant for a limited time interval.

As discussed above, the PREMOS and SIM measurements agree 
well for sunspot passages (with irradiance variations from $1000 \mathrm{ppm}$ to $-600 \mathrm{ppm})$. They also agree within their uncertainties for time periods dominated by enhanced plages $(>600$ $\mathrm{ppm})$. However, they differ significantly for intermediate irradiance variations from $-400 \mathrm{ppm}$ to $-200 \mathrm{ppm}$ and close to $+300 \mathrm{ppm}$. The reason for this discrepancy is not obvious. Also, the PREMOS distribution is more Gaussian compared to that for SIM, as shown in Fig.17. A possible explanation would be a higher intrinsic noise level in PREMOS data, both widening the distribution and making it more Gaussian.

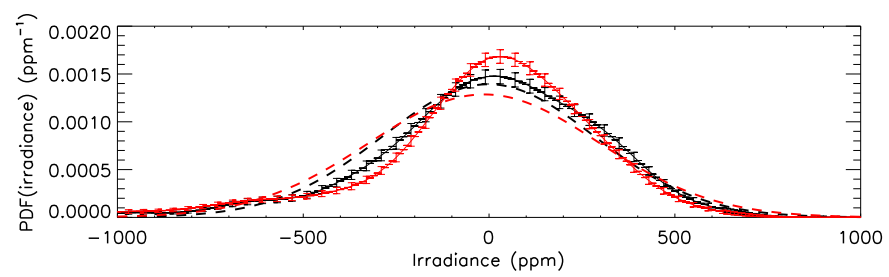

Fig. 17. Probability distribution function of the irradiance as observed by PREMOS (in black) and SIM (in red) at $535 \mathrm{~nm}$ with their uncertainties and with the respective Gaussian fit (dashed line).

A similar approach has been used to study the irradiance at 607 and $782 \mathrm{~nm}$ as measured by PREMOS. Figures 18 and 19 show the corresponding curves for 607 and $782 \mathrm{~nm}$, respectively, from July 2011 to July 2012. As for $535 \mathrm{~nm}$, the full time series for $607 \mathrm{~nm}$ and $782 \mathrm{~nm}$ are presented in Appendix A. However, PREMOS data at $607 \mathrm{~nm}$ after September 2013 are not considered because of a high uncertainty. The noise level in the PREMOS observations has been estimated in the same way as for $535 \mathrm{~nm}$, and is summarised in Table 5. An envelope of about $\pm 245 \mathrm{ppm}$ and $\pm 125 \mathrm{ppm}$ has been added to the PREMOS observations for $607 \mathrm{~nm}$ and $782 \mathrm{~nm}$, respectively. As for $535 \mathrm{~nm}$, there is a good overall correlation when taking the noise level of PREMOS data into account, despite rather low correlation coefficients (see Table 5).

Table 5. Number $\mathrm{N}$ of common days shared by the full PREMOS time series with SORCE observations for the visible and near-infrared channels, along with $\mathrm{R}$ and $\mathrm{R}^{2}$ (in brackets) values, and the noise level data within the different PREMOS channels.

\begin{tabular}{cccc}
\hline & $\mathrm{N}$ & $\mathrm{R}\left(\mathrm{R}^{2}\right)$ & $\sigma$ (in ppm) \\
\hline $535 \mathrm{~nm}$ & 858 & $0.62(0.39)$ & 210 \\
$607 \mathrm{~nm}$ & 636 & $0.57(0.32)$ & 245 \\
$782 \mathrm{~nm}$ & 835 & $0.59(0.35)$ & 125 \\
\hline
\end{tabular}

\section{Conclusions}

In this paper, we have presented the in-flight performance of the PREMOS instrument aboard the PICARD satellite. For nearly three and a half years, PREMOS demonstrated its ability to produce time series of solar irradiance from the UV spectral range (observed at $210 \mathrm{~nm}, 215 \mathrm{~nm}$, and $266 \mathrm{~nm}$ ) to the visible and near-infrared spectral ranges (with observations at $535 \mathrm{~nm}, 607$ $\mathrm{nm}$, and $782 \mathrm{~nm}$ ). We presented different correction procedures, leading to the definition of Level $2 \mathrm{~B}$ data, where the temperature
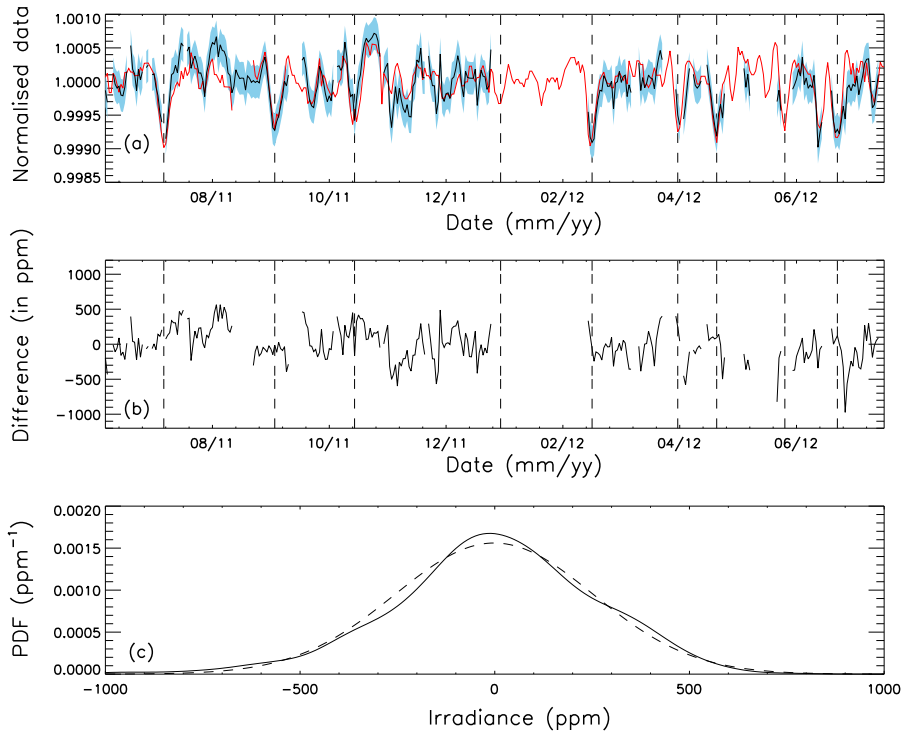

Fig. 18. Same as in Fig.16 except for irradiance at $607 \mathrm{~nm}$.
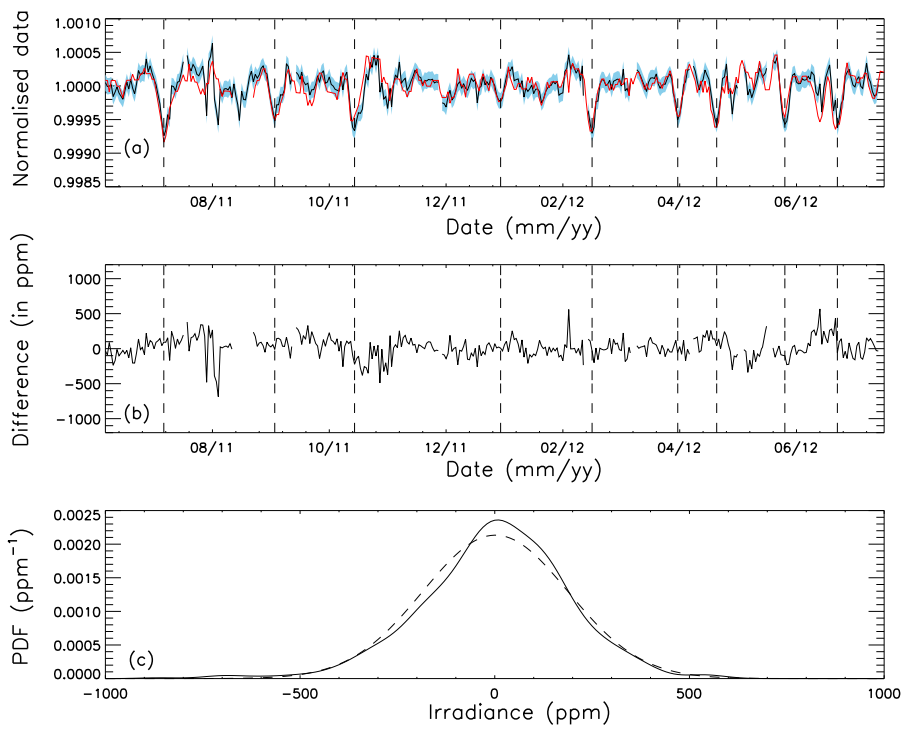

Fig. 19. Same as in Fig.16 except for irradiance at $782 \mathrm{~nm}$.

dependencies and non-solar features are extracted, and definition of the Level 3 data, where a correction for degradation was applied.

For the channels at $210 \mathrm{~nm}$ and $266 \mathrm{~nm}$, a proxy-based model was used to correct the long-term degradation (i.e. over the PREMOS lifetime). Regarding the irradiance at $215 \mathrm{~nm}$, the degradation correction was conducted using only the backup channel, which was exposed once per week. The Level 3 PREMOS data in the UV were then compared to the SORCE/SOLSTICE observations, and excellent agreement was found with a ratio always less than $1 \%$. One important outcome of the PREMOS experiment clearly is the result regarding the $215 \mathrm{~nm}$ channel. The degradation of this UV channel was corrected using one measurement every week over a period of nearly three years. It is difficult to reach any conclusions over longer periods since no external data sets are available for comparison. 
The PREMOS observations in the visible and near-infrared are only corrected for degradation over the short-term. More efforts, especially using PICARD/SODISM and PROBA2/LYRA data, are required to understand the particular degradation behaviour over the long-term. For the short-term only, we performed a comparison with SORCE/SIM observations. Taking into account the intrinsic noise level with SIM, we estimated the signal-tonoise ratio for the PREMOS channels. We find the data sets are in good agreement, with a noise level from $125 \mathrm{ppm}$ to $245 \mathrm{ppm}$. Finally, high cadence data in the visible and near-infrared have been tested in a helioseismologic context, leading to the identification of the p-mode. The signature of the Venus transit has led to the definition of the intrinsic noise level within the high cadence channels.

Level 3 data have successfully been tested leading to a validated PREMOS data set, which can be used to address several scientific topics. Some have already been discussed here, namely the detection of flares. However, more analysis related to the Venus transit can be performed by looking at the small-scale structures on the solar surface. We also mentioned the possibility of using PREMOS data to fill data gaps in the UV. The PREMOS data from the UV to the visible and near-infrared spectral ranges could also be used to validate semi-empirical models of the solar irradiance (see e.g. Cessateur et al. 2016b). Level 3 data, as presented in this paper, will be available through the MEDOC database $^{2}$ and through the SOLID database ${ }^{3}$, while Level 2B data are available on request.

Acknowledgements. This study received funding from the European Community's Seventh Framework Programme (FP7/2007-2013) under the grant agreement nr. 284461 (eHEROES project, http://soteria-space.eu/eheroes/html/) and nr. 313188 (SOLID project, http://projects.pmodwrc.ch/solid/). GC, TE, and ER acknowledge support from the Swiss COST office (grant nr. C11.0135). GC, $\mathrm{MH}, \mathrm{AS}, \mathrm{ER}, \mathrm{TE}$, and WS benefited from the participation in the COST action ES1005 (TOSCA) meetings. MK acknowledges funding by the CNES/PICARD GI programme "Analysis of spectral irradiance observed by PREMOS". PICARD is a mission supported by the Centre National d'Etudes Spatiales (CNES), the Centre National de la Recherche Scientifique (CNRS/INSU), the Belgian Space Policy (Belspo), the Swiss Space Office (SSO), and the European Space Agency (ESA). The PREMOS instrument is supported by the Swiss PRODEX for the engineering, design, and hardware costs. The authors wish to thank the referee for careful reading of the manuscript and fruitful comments and suggestions

\section{References}

Barthelemy, M. \& Cessateur, G. 2014, Journal of Space Weather and Space Climate, 4, A35

BenMoussa, A., Dammasch, I. E., Hochedez, J.-F., et al. 2009, A\&A, 508, 1085

BenMoussa, A., Gissot, S., Schühle, U., et al. 2013, Sol. Phys., 288,389

Buisson, F., Meissonnier, M., Mouret, J., et al. 2006, in Proceedings of the 4S Symposium: Small Satellites, Systems and Services, Chia Laguna Sardinia, Italy, 25 - 29 Sept, ed. D. Danesy, ESA SP-625

Cebula, R. P., DeLand, M. T., \& Hilsenrath, E. 1998, J. Geophys. Res., 103, 16235

Cessateur, G., Barthelemy, M., \& Peinke, I. 2016a, Journal of Space Weather and Space Climate, in press, DOI: $10.1051 / \mathrm{swsc} / 2016009$

\footnotetext{
${ }^{2}$ http://idoc-picard.ias.u-psud.fr/sitools/client-user/Picard/projectindex.html

${ }^{3}$ http://projects.pmodwrc.ch/solid/
}

Cessateur, G., Dudok de Wit, T., Kretzschmar, M., et al. 2011, A\&A, 528, A68

Cessateur, G., Kretzschmar, M., Dudok de Wit, T., \& Boumier, P. 2010, Sol. Phys., 263, 153

Cessateur, G., Shapiro, A., Yeo, K., et al. 2016b, A\&A, submitted

Conscience, C., Meftah, M., Chevalier, A., Dewitte, S., \& Crommelynck, D. 2011, SPIE Conference Series, 8146, 13

Corbard, T., Salabert, D., Boumier, P., et al. 2013, Journal of Physics Conference Series, 440, 012025

Domingo, V., Ermolli, I., Fox, P., et al. 2009, Space Science Reviews, 145, 337

Dominique, M., Hochedez, J.-F., Schmutz, W., et al. 2013, Sol. Phys., 286, 21

Dudok de Wit, T., Kretzschmar, M., Lilensten, J., \& Woods, T. 2009, Geophys. Res. Lett., 36, 10107

Dudok de Wit, T., Lilensten, J., Aboudarham, J., Amblard, P., \& Kretzschmar, M. 2005, Annales Geophysicae, 23, 3055

Egorova, T., Rozanov, E., Manzini, E., et al. 2004, Geophys. Res. Lett., 31, 6119

Ermolli, I., Matthes, K., Dudok de Wit, T., et al. 2013, Atmospheric Chemistry \& Physics, 13, 3945

Fröhlich, C. 2013, Space Sci. Rev., 176, 237

Fröhlich, C., Romero, J., Roth, H., et al. 1995, Sol. Phys., 162, 101

Haberreiter, M., Schmutz, W., \& Hubeny, I. 2008, A\&A, 492, 833

Harder, J. W., Fontenla, J., Lawrence, G., Woods, T., \& Rottman, G. 2005, Sol. Phys., 230, 169

Harder, J. W., Fontenla, J. M., Pilewskie, P., Richard, E. C., \& Woods, T. N. 2009, Geophys. Res. Lett., 36, 7801

Hochedez, J., Schmutz, W., Stockman, Y., et al. 2006, Advances in Space Research, 37, 303

Kretzschmar, M. 2011, A\&A, 530, A84

Kretzschmar, M., Dominique, M., \& Dammasch, I. E. 2013, Sol. Phys., 286, 221

Kretzschmar, M., Dudok de Wit, T., Schmutz, W., et al. 2010, Nature Physics, 6, 690

Lilensten, J., Dudok de Wit, T., Kretzschmar, M., et al. 2008, Annales Geophysicae, 26, 269

Meftah, M., Dewitte, S., Irbah, A., et al. 2014a, Sol. Phys., 289, 1885

Meftah, M., Hochedez, J.-F., Irbah, A., et al. 2014b, Sol. Phys., 289,1043

Rottman, G. 2005, Sol. Phys., 230, 7

Rottman, G., Floyd, L., \& Viereck, R. 2004, in Washington DC American Geophysical Union Geophysical Monograph Series, Vol. 141, Solar Variability and its Effects on Climate. Geophysical Monograph 141, ed. J. M. Pap, P. Fox, C. Frohlich, H. S. Hudson, J. Kuhn, J. McCormack, G. North, W. Sprigg, \& S. T. Wu, 111

Schmutz, W., Fehlmann, A., Finsterle, W., Kopp, G., \& Thuillier, G. 2013, AIP Conference Proceedings, 1531, 624

Schmutz, W., Fehlmann, A., Hülsen, G., et al. 2009, Metrologia, 46, S202

Schöll, M., Dudok de Wit, T., Kretzschmar, M., \& Haberreiter, M. 2016, Journal of Space Weather and Space Climate, in press

Scott, D. W. 1992, Multivariate Density Estimation: Theory, Practice, and Visualization (New York, Chicester: John Wiley and Sons)

Shapiro, A. I., Schmutz, W., Dominique, M., \& Shapiro, A. V. 2013, Sol. Phys., 286, 271 
Shapiro, A. I., Schmutz, W., Schoell, M., Haberreiter, M., \& Rozanov, E. 2010, A\&A, 517, A48

Shapiro, A. V., Rozanov, E., Shapiro, A. I., et al. 2012, Atmospheric Chemistry \& Physics, 12, 3181

Snow, M., McClintock, W. E., Rottman, G., \& Woods, T. N. 2005, Sol. Phys., 230, 295

Thuillier, G., Dewitte, S., Schmutz, W., \& The Picard Team. 2006, Advances in Space Research, 38, 1792

Thuillier, G., Foujols, T., Bolsée, D., et al. 2009, Sol. Phys., 257, 185

Thuillier, G., Schmidtke, G., Erhardt, C., et al. 2014, Sol. Phys., 289,4433

Wehrli, C., Schmutz, W., \& Shapiro, A. I. 2013, A\&A, 556, L3

Yeo, K. L., Krivova, N. A., Solanki, S. K., \& Glassmeier, K. H. 2014, A\&A, 570, A85

\section{Appendix A: Full PREMOS time series for the visible and near-infrared spectral ranges}

The time series for $535 \mathrm{~nm}, 607 \mathrm{~nm}$, and $782 \mathrm{~nm}$, from October 2010 to December 2013 are shown in Figures A.1 to A.3. The analysis of those curves is presented in Sect. 4.2.2. 

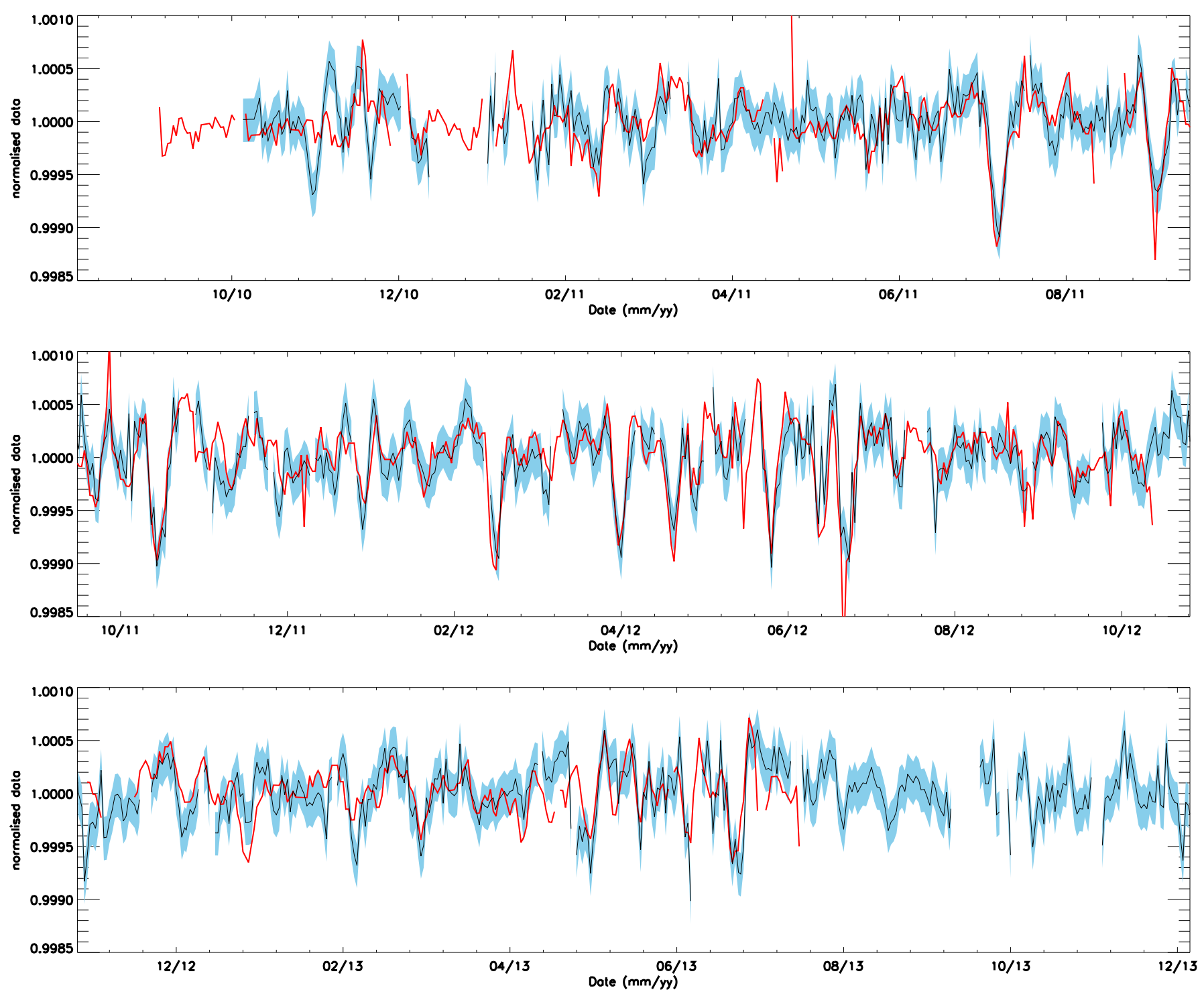

Fig. A.1. Short-term variability of the irradiance at $535 \mathrm{~nm}$ as observed by PREMOS (in black) and SORCE/SIM (in red) from October 2010 to December 2013. 

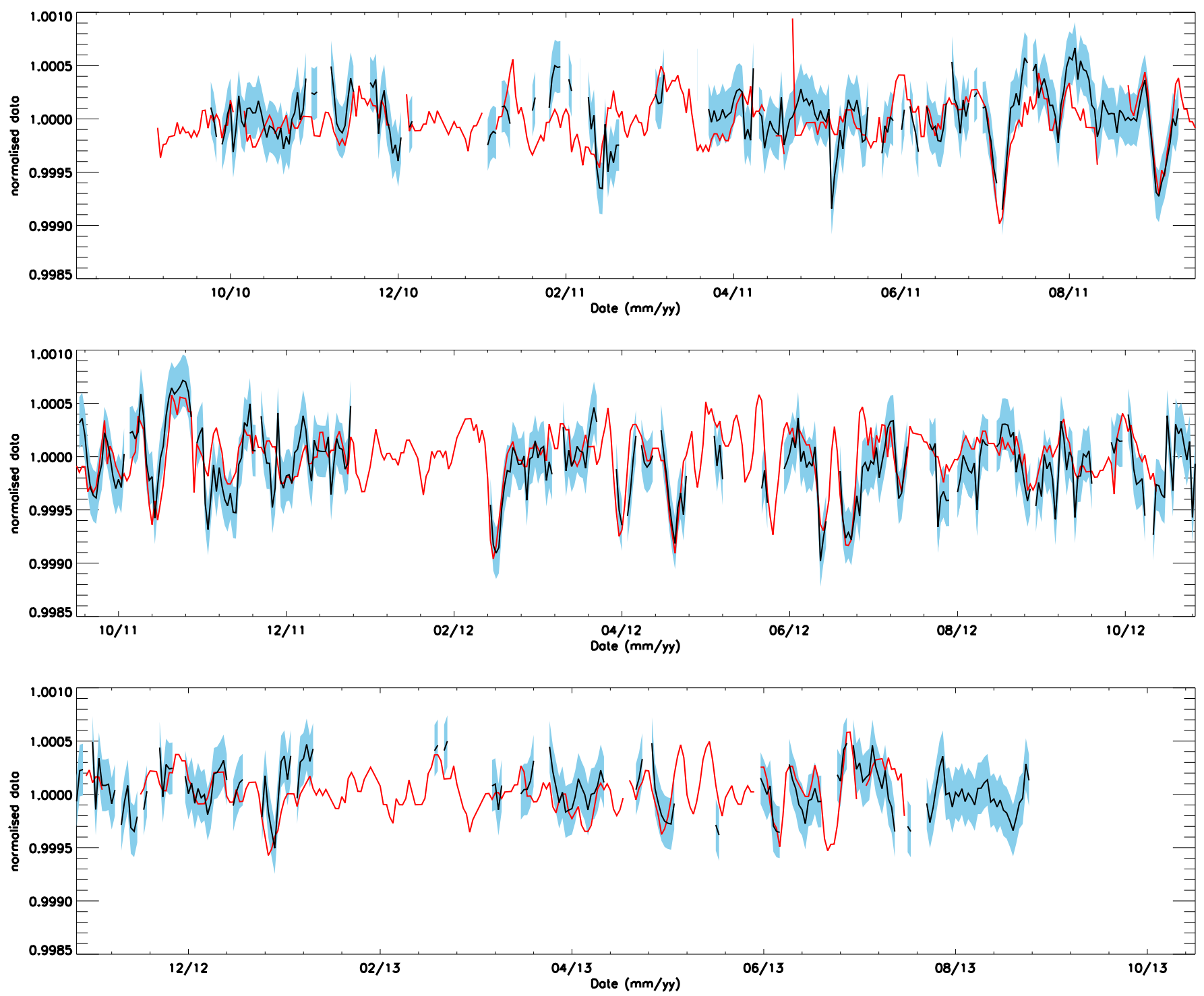

Fig. A.2. Same as in Fig. A.1 except for irradiance at 607 nm from October 2010 to September 2013. 

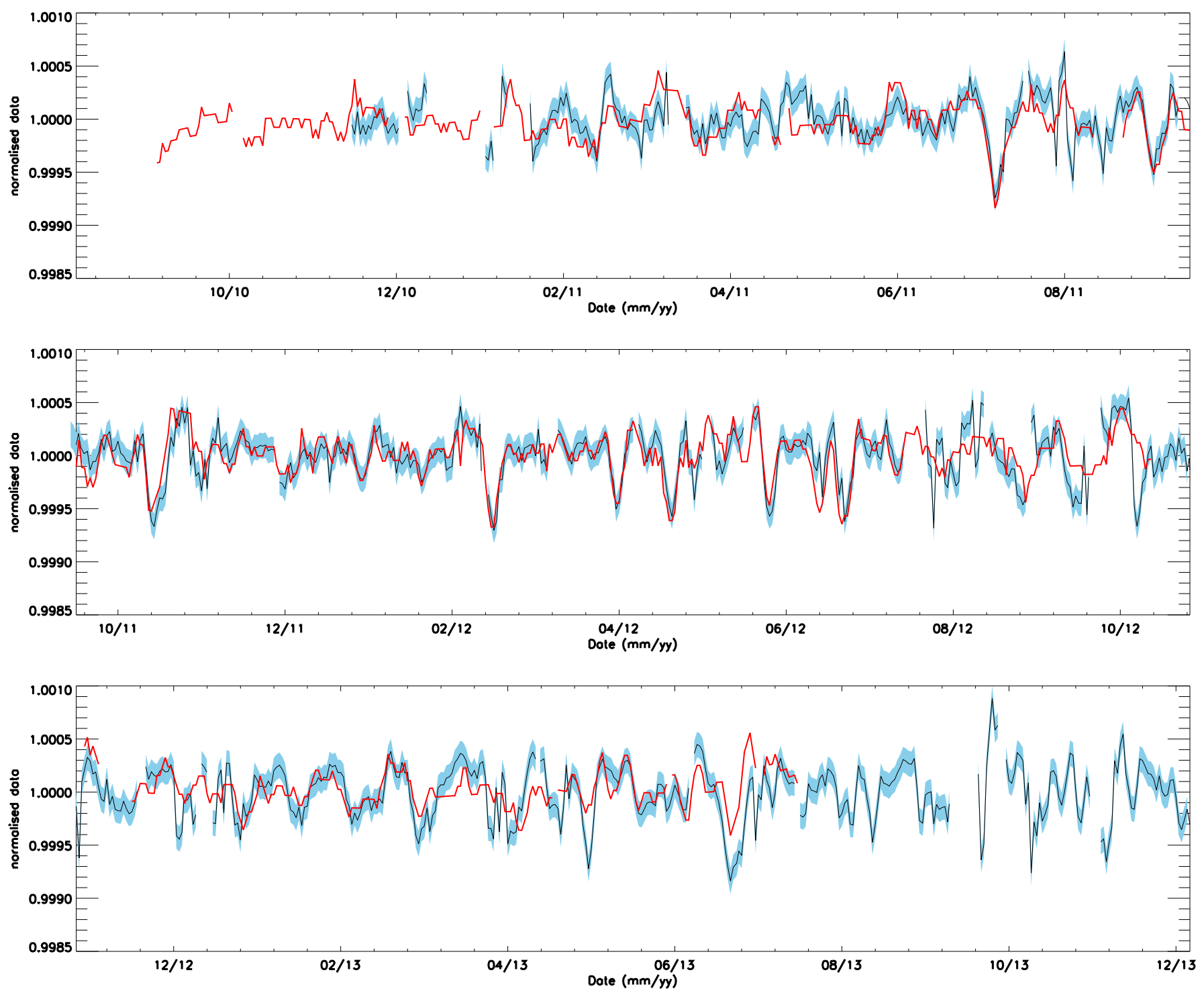

Fig. A.3. Same as in Fig. A.1 except for irradiance at $782 \mathrm{~nm}$. 